\title{
Intelligent business processes composition based on multi-agent systems
}

\author{
José A. García Coria ${ }^{\mathrm{a}}$, José A. Castellanos-Garzón ${ }^{\mathrm{b}}$, Juan M. Corchado ${ }^{\mathrm{c} *}$ \\ Department of Computers and Automation, University of Salamanca, Plaza de la Merced $s / n, 37008$ \\ Salamanca, Spain
}

\begin{abstract}
This paper proposes a novel model for automatic construction of business processes called IPCASCI (Intelligent business Processes Composition based on multi-Agent systems, Semantics and Cloud Integration). The software development industry requires agile construction of new products able to adapt to the emerging needs of a changing market. In this context, we present a method of software component reuse as a model (or methodology), which facilitates the semi-automatic reuse of web services on a cloud computing environment, leading to business process composition. The proposal is based on web service technology, including: (i) Automatic discovery of web services; (ii) Semantics description of web services; (iii) Automatic composition of existing web services to generate new ones; (iv) Automatic invocation of web services. As a result of this proposal, we have presented its implementation (as a tool) on a real case study. The evaluation of the case study and its results are proof of the reliability of IPCASCI.
\end{abstract}

Keywords: Multi-agent system, Web service, Cloud computing, Software component reuse, Software engineering, Business process composition, Machine learning.

*Corresponding author. E-mail address: $\left\{\right.$ jalberto $^{a}$, jantonio $^{b}$, corchado $\left.^{c}\right\} @$ usal.es

Preprint submitted to Expert Systems with Applications

August 292013 


\section{Introduction}

The software development industry is constantly evolving and looking for new technologies, languages and tools that are increasingly powerful, efficient and safe. In this process, it is essential to build models, architectures and agile technologies able to introduce new tools as simply and economically as possible. Component reuse is actually one of the techniques that more clearly contribute to such a development by providing efficient mechanisms to create quality software [1-6]. Reuse increases software reliability (because it uses tested software components), development productivity and implies a clear cost reduction [6-8]. Since the recent increasing of the volume and complexity of software products, reuse has been a field taken into great consideration, being a fundamental stage in design and quality models as CMMI (Capability Maturity Model Integration) [9].

In this context, web service reuse appears as an interesting alternative with respect to code classical reuse. Indeed, a web service is a software component representing a service deployed on a web platform and supporting automatic interactions between machines on a computer network [10]. In this framework, arises SOA (Service-Oriented Architecture) as a new architecture leading to conventional software development [11-13]. SOA introduces a new method to create distributed applications where their basic services can be discovered, published, and linked in order to achieve more complex services. Applications interact through the existing services from entry points on an interface rather than at the level of implementation [14].

Software development can be analyzed from different perspectives, offering us a wide variety of alternatives to a methodological, functional and instrumental level, among others. One of the paradigms revolutionizing this industry in recent years has been cloud computing [15-19]. The cloud represents a novel concept of service and information distribution, providing many possibilities of scaling solutions, facilitating the use of relatively simple and economic terminals (interesting models of pay per use, and multi-platform accessibility, etc). However, this model has certain drawbacks related to maintenance complexity and application development [20, 21]. Moreover, the number of experienced engineers in this field is relatively low and the development time is significantly high [22-24]. 
Starting from all the above, this research proposes a methodology (IPCASCI, Intelligent business Processes Composition based on multi-Agent systems, Semantics and Cloud Integration) that facilitates the business process construction on cloud computing environments in an agile and efficient way from developed components. It deals with the development of a proposal that facilitates the creation of such processes in form of web services from other semi-automatic functional services. All this is carried out on the framework of a computer system guided by relatively inexpert programmers. The process of business process construction is guided by a multi-agent architecture based on virtual organizations [25-27], which is able to implement the intelligent behavior needed for process management by using ontology [28-32]. The goal of this research is then to provide a model allowing the automatic construction of a business process from specifications in text format (with some constraints) of the process concerning us. The multi-agent system based on virtual organizations will facilitate the process composition by using standard BPEL (Business Process Execution Language). This standard allows the automatic composition of $w e b$ services in an easy way by adding the advantage of a direct projection to a diagram BPMN (Business Process Management Notation) [33].

To reach the goals proposed in this research, the remainder of the present paper has been structured into the following sections: Section 2 outlines information and works related to this research. Section 3 deals with an overview of the different components coupled to our proposal to make the web service composition, that is, architecture IPCASCI. This section gives a general idea of the whole composition process from IPCASCI. Section 4 presents cloud computing and web service technology, which have been included in our proposal. On one hand, we describe the advantages of developing IPCASCI on a cloud environment and provide two cloud functionalities to support its performance. On the other hand, we introduce web services technology and explain the inclusion of semantics to the web services through ontology to later facilitate the process of discovery and composition from a multiagent system. Section 5 describes the agent-based virtual organizations defined in the multiagent system of IPCASCI to make the discovery of the web services fit the specifications of the user to compose them and obtain the solution web service. Section 6 develops a real case 
study to give an implementation of IPCASCI, which is evaluated through software metrics. Conclusions, Appendix A with information supporting our proposal and the references of this research have been given at the end of this paper.

\section{Related work}

As previously explained, we have introduced an architecture (IPCASCI) aimed at composing web services to obtain new ones in an automatic way by starting from requirements given by the user. This process is carried out in such a way that the service automatic construction be efficient and has an intelligent behavior. Intelligent behavior is intended as the ability of processing of the input requirements introduced by the user, that is, the system be able to: (i) analyze the input, (ii) find the web services allowing to hold the requirements, and (iii) carry out an automatic composition of web services for their corresponding business processes. Thus, as a final result, new web services implementing the provided requirements will be achieved in an automatic process of discovery and composition. In this sense, there exist several approximations of architectures based on semantic web services. In [34], a solution focused on fuzzy logic to discover semantic web services has been proposed. In [35], a solution based on agents and ontological language DAML-S has been proposed, and in [36], a proposal based on queries SPARQL and ontological language OWL-S has also been used. In general terms, most of the proposals on architectures of semantic web services are based on language OWL-S. The proposal given in this paper differs from the existing ones in the following:

- Design of a global platform, which is embedded in a cloud environment and whose structure has been provided for an agile and efficient running.

- The semantics information of the web services does not depend on the internal structure of the ontology, which allows reusing ontologies regardless of their formats.

- We have included a multi-agent system based on virtual organizations that facilitates requirement analysis of the user and the discovery process of web services. 
- The solution web service has been built from a simple definition given through a set of specifications introduced by the client.

\section{Architecture IPCASCI for business process composition}

This section describes the different components integrated to our proposal IPCASCI, which allow carrying out the automatic composition of web services. So Figure 1 shows a conceptual diagram of the components integrating the proposed architecture. This figure describes the following items:

1. Cloud system: Platform of cloud computing on which is supported our proposal. The platform provides an environment for running and storage.

2. Web services: The web services existing in the architecture that will be used by the process of web service composition.

3. UDDI register: Universal register system where used web services are registered. This allows us an open access to the web services of the architecture. This way, the web services implemented on the architecture may be reused regardless of our proposal.

4. Multi-agent system based on virtual organization: This system supports functionalities to make the discovery and the composition of web services:

a. Analysis system: Analyze the semantic content introduced by the user in order to structure it in computable items.

b. Search system: Discover the web services that hold the semantics as syntactic constraints given by the user.

c. Composition system: Once determined the web services and their relationships holding the user semantic requirements, a service composition is applied on such web services to obtain the new web service.

5. Ontologies: Distinct ontologies modeling the semantic knowledge that can be included in the web services.

6. BPEL file: Store the composition of the web services that meet the requirements indicated by the user to obtain the desired solution. 
As a general schema of working, IPCASCI accepts the requirements of the user through the assistant software to raise the processes of discovery and composition. The steps followed by IPCASCI have been given the following algorithm and represented in Figure 2:

\subsection{Algorithm for web service discovery and composition (AWSDC).}

1. The user introduces the requirements of the web service to build by means of an assisted system. Such a system allows users to define a list of terms (in form of modules) representing the requirements of the new web service. The list of terms is bounded by the web service repository in the cloud system. The result of this process is a set of related modules (analysis system) in such a way that each module registers the following information:

a. Name;

b. Input;

c. Output;

d. Process carried out by the module (semantic concept it represents);

e. Domain of the semantic concept represented by the module (ontological domain);

f. Preconditions of running;

g. Relationship with other modules.

2. For each module of the above step, an automatic search (search system) for the $w e b$ services that include the requirements of the module is carried out. Moreover, the search is made in syntactic and semantics form.

3. From the information of the above step, a reduced BPD diagram (Business Process Diagram, [37]) is built through of graphic standard BPMN (Business Process Management Notation), on which each web service is represented as an activity and also showing the interactions existing between the web services. 
4. The BPD diagram is then displayed by the assistant software to the user, because could exist several web services implementing a single activity. In this case, the user can select another web service different from the one given by default in the diagram.

5. From the BPD diagram, a composition BPEL (Business Process Execution Language, [38]) is carried out in such a way that the service specified by the user is built and can later be invoked (composition system) as one more service in the platform. See Figure 2 and 3 to summarize the whole process.

As shown in Figure 3, the assistant software interprets the requirements introduced by the user (the requirements are restricted to the ontological domain defined on the addressed problem) to build the new web service. These requirements are later processed by IPCASCI in the cloud system, which runs a multi-agent system based on virtual organizations to discover and compose the web services including the requirements of the user. As a result, a BPMN (Business Process Management Notation) solution is obtained which represents, through activities, the functionalities of the required web service. Such a solution can be fixed by the user to confirm the diagram. Finally, the new web service is generated and included in the platform, so that users/clients can use it.

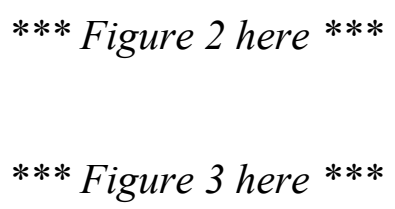

Note that with respect to standards BPMN and BPEL, we have that the first one specifies a diagram of business process (BPD), offering several advantages [37] as modeling business processes in a simple way, to be readable by nontechnical users, providing tools to model complex business processes and simple mapping to business execution languages as BPEL. This way, BPEL is the standard to provide business process specifications for a web service 
environment. It allows composing web services, representing them as an operation flow in a diagram, and states such a composition as a new web service.

\section{Cloud computing and web services in IPCASCI}

This section describes the functionalities provided by the cloud system and web service technology coupled to architecture IPCASCI. The idea is to give a more detailed description of the associated points of the algorithm presented in the above section needing further background.

\subsection{Cloud system}

We have defined an architecture for web service composition on a cloud computing environment, which implies that the software generated from it will be of shared and distributed use. Moreover, its management should be efficient, reliable and the access to it should be from any platform $[22,24,39]$. These characteristics provide the development of scalable, dynamic and distributed software, representing advantages on the current distributed systems [40]. All this, allows us integrates components as a multi-agent system and SOA (service-oriented architecture, [11-13]) to our architecture [41, 42].

According to the above, the services offered by the proposed model through cloud computing are focused on the layers of infrastructure and software. The infrastructure layer provides services as file storage, which relieves the client for the liability of acquiring the corresponding storage systems and maintenance. On the other hand, the software layer provides a set of services REST [43], allowing managing operations for automatic discovery and composition of web services. Particularly, the proposed model provides two functionalities, namely: analyze\&Discover and composeService. The first functionality accepts as input a XML document (Extensible Markup Language, [44]) representing the requirements of the user for which will be generated a diagram according to standard BPMN that models the input, Figure 4. For each activity of the diagram, there is a set of web services fixed the semantic and syntactic requirements of the concept 
represented by the activity (module/concept introduced by the user). Therefore, the output of this functionality is another XML document representing a mapping of the input to a BPMN graphic. The second functionality accepts as an input a diagram BPMN in a XML document, which is a reduced diagram, that is, each activity of the diagram is associated with a single web service. From this input, the composition of the web services associated with each activity will be carried out to finally build the required web service by means of BPEL (Business Process Execution Language). Note that both functionalities encapsulate the multi-agent system for discovering web services and the composition of such services, respectively. This allows us an abstraction level from the different components coupled to the architecture from the implementation point of view.

\section{*** Figure 4 here ***}

\subsection{Web services and inclusion of semantics}

The main goal of our proposal is web service reuse for its composition in order to generate new web services, automatically. Starting from this, we have focused on SOA technology (Service Oriented Architecture, [45]) to represent web services by means of description WSDL, register UDDI, messaging common protocols (SOAP). The choosing of web service reuse is due to its modular nature uncoupling the interface of the service from the implementation, and the ability of linking dynamically services.

As previously explained, the engine of web service discovery and analysis has been given by a multi-agent system (MAS), for which has been developed several works integrating it to SOA technology $[46,47]$. Hence, we integrate web services and SOA following the diagram in Figure 5. As shown in this figure, the MAS represents the ontology concepts (modeled by an ontology defined in box Ontologies, $[48,49])$ that will include the new web service, and is linked to register UDDI where the web services of the platform have been published. The description (WSDL files) of such web services has been given in box WSDL, which has been linked to box Ontologies, implying that each description WSDL of web services 
includes the ontological concept it represents. Note that the inclusion of ontology to represent concepts is the basis of the discovery and analysis process of web services (machine learning). In this sense, the MAS given in Figure 5 creates an agent for each ontological concept to assist the discovery process of web services, which is based on semantic concepts.

Based then on the above, we have used language WSDL-S (Web Service Semantics, [50]) to make semantics annotations on descriptions WSDL of the web services. WSDL-S introduces an association mechanism of semantics annotations for web services described using WSDL. Thus, WSDL-S defines a domain ontology on web services. This means that the ontology provides labels to add semantics to web services through insertions (annotations) into files WSDL [51, 52], and includes three sub-ontologies for this purpose, that is: service profile, service grounding and process model. Summarizing, WSDL is the means of description for web services. On the basis of its descriptive ability, a mechanism to annotate abilities and requirements referenced from a semantic model (ontology WSDL-S) has been provided. Such characteristics allow the architecture to automate the discovery process of web services (semantic matching of user requirements with web services). Hence, we use a MAS to model the ontology, which create agents representing each ontological concept, connecting them (communication lines between agents) by equivalent concepts. In addition, each agent also builds a list of links to entries to the UDDI register, whose web services include the concept it represents, as shown in Figure 6.

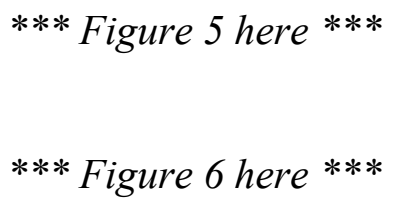

\section{Multi-agent sytem (MAS) for service discovery and composition in IPCASCI}

This section outlines the structure and performance of the MAS involved in our architecture. As explained in Section 2 and now shown in Figure 7, this MAS consists of three agent-based virtual organization subsystems, which have been classified in analysis, 
search and composition system. These systems have been built in form of black box whereby, they do not depend on other systems, and there are not communication lines between them. Hence, any commutation between them is scheduled by agent manager. This way, these agent-based virtual organizations can apart be modified or adapted without affecting the performance of the remaining systems in the architecture.

$$
\text { *** Figure } 7 \text { here } * * *
$$

\subsection{Analysis system}

The analysis system manages the requirements introduced as input by the user. It processes the requirements by converting them into computable information and achieving enough semantics information to later carry out a search and composition of web services. This system is integrated by a main agent called analysis coordinator. The requirement input of the user has been made through a graphic assistant (software) that integrates each generated module. We have introduced the module concept to encapsulate the attributes representing a semantic concept, and it has been defined on the following characteristics:

1. Functionality: It describes the functionality to be carried out by the module and is introduced in text format by means of a list of semantic concepts associated with the module. Optionally, it is possible to include preconditions to hold by the module before the running.

2. Domain: From the definition of each module the assistant allows the user to choose the domain name of a list of existing domains. The domain classifies the workspace where we are going to perform the task and the ontology to use.

3. Input: The user determines the number of inputs of the module based on the concept it represents.

4. Output: By a similar way as the input, the user specifies the output generated by the module. 
5. Interconnection: The assistant guides the user to define the connection of the current module with the previously defined modules. In this sense, the following instructions of flow control to connect modules have been defined to represent the information given by the user:

a. [Module_i] If [Condition] then [Module_j]

b. [Module_i] If [Condition] then [Module_j] else [Module_k]

c. [Module_i] Parallel output to [Module_j], [Module_k]

d. [Module_i] Follows [Module_j]

When the user completes the module definition and their relationships, the assistant builds a flow diagram with computable information aimed at generating the new web service and so the assistant stores the diagram in an XML document and passes to the next step. The assistant then invokes cloud functionality analyze\&Discover (see Section 4.1), having as an input the flow diagram in XML format. Thus, this functionality runs the analysis system (and later runs the search system) of the architecture by passing a message to the manager agent to run the analysis coordinator agent, Figure 8. Hence, the analysis coordinator agent is responsible for interpreting the information of the XML document (by running a parser) to create an agent-based virtual organization representing the flow diagram saved in XML format by the assistant. To this end, each built agent by this virtual organization has been associated with a single module in the input XML document, representing in that way, the semantic concept assigned to such a module. Between the agents of this new virtual organization have also been created communication lines (conditional flow control) based on the module relationships described in the diagram given by the XML document. So that the flow diagram has been modeled as a graph, where nodes are agents, edges are communication lines and the whole graph represents the domain ontology. Then, each agent in this virtual organization manages input, output and description (preconditions and effects) for the module it represents, Figure 9. Moreover, as explained in the next subsection (search system), each agent will also be responsible for creating a list of links to the UDDI register for the web services that include the ontological concept it represents. 
Completed the construction of the agent subsystem to represent the domain ontology, diagram BPD (Business Process Diagram) based on graphic standard BPMN (Business Process Management Notation) will be generated. In this case, every activity in the diagram has been associated with an agent of the ontology, and the flow control lines of the diagram have also been associated with the communication lines between the agents. All this process is intended to store diagram BPD to later convert it into a BPEL file (Business Process Execution Language), which will be run to build the expected web service. Basically, the transformations to convert the agent subsystem into a diagram BPD have been given in Table 1.

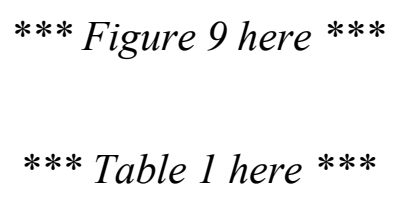

In Section A.1 of Appendix A, an example of converting the agent subsystem into the BPD diagram has been given by basing on Table 1. As shown in Figure A2 of this example, the BPD diagram has been obtained from the agent subsystem given in Figure A1. In the next step, it would be generated an XML document (BPEL file) with the structure of the diagram in Figure A2 and the semantics information early given by the user. Note also that each activity on the BPD diagram will later be associated with the list of web services implementing the semantic concept (a web service of the list has been assigned by default) it represents by running the search system.

\subsection{Search system}

The search system is responsible for finding a list of web services from the semantic concepts and their syntaxes received from the manager agent. That is, a discovery process of 
web services will be carried out through semantic matching. That is, a search for the web services whose semantics annotations on theirs descriptions WSDL match the ones previously defined in the modules. Thus, functionality analyze\&Discover also runs the search system by passing diagram BPD (XML document) that the analysis system has built through the manager agent. The search system will also complete such a diagram BPD with the web services found. Then, the discovery process has been carried out once for each agent of the agent subsystem created by the analysis system. Thus, each agent in this subsystem invokes its skill discover to achieve a list of web services representing its semantics description. Figure 10 shows the agents integrating the search system and their connections.

As displayed in this figure, the search system consists of several agents responsible for reaching the following aims:

1. Search coordinator: It receives as input the description of a module (from the agent representing the concept associated with the module), which is provided by the manager agent. Therefore, the search coordinator agent is responsible for coordinating the whole search process of web services. It returns as a result, a list of web services that fits the input description.

2. Semantic coordinator: It receives the semantic concepts that most include the web services from the search coordinator agent. Then, for each concept, it sends a message to the localizer agent (that belongs to the register subsystem), which returns a list of links to register UDDI with the web services implementing such concepts. Note that through of this process the semantic matching is carried out from the concepts processed by the semantic coordinator agent and the semantics annotations previously made on files WSDL of the web services.

3. Checking coordinator: It receives the WSDL file of a web service and the conditions to be fulfilled by the service from the search coordinator agent. Then, it communicates with the checking subsystem, which determines whether such a web service holds the given syntactic requirements. Finally, it returns the checking result to the search coordinator agent. 
Summarizing on the search system, we have that its first part consists of finding the web services associated with a semantic content. To do this, the search coordinator agent communicates with the semantic coordinator agent, who requests to the localizer agent (which is based on register subsystem), the list of links to the UDDI register for the web services that include the given concept. This process is done once for each provided concept. Then, when the semantic coordinator agent has found the web services associated with each concept, it carries out a filtering process to extract the services that implement all concepts. These last web services will be returned to the search coordinator agent as shown in Figure 11. Note that the inclusion of attribute domain in the ontological concepts classifies the web services into groups in such a way that the semantics search is made only on a specific group of services that match their domain, which improves the runtime of the search. Finally, the search coordinator agent passes the list of web services to the checking coordinator agent, which filters, through the checking subsystem, the ones that hold the syntactic constraints. The final list of web services associated with the given concept is assigned to the activity representing such a concept in diagram BPMN (BPD).

$$
\text { *** Figure } 11 \text { here } * * *
$$

\subsubsection{Register subsystem}

As previously explained, the ontological information given by the user has been mapped on an agent subsystem, that is, a graph of interconnected agents. In this sense, the localizer agent of the register subsystem in Figure 10 sends a broadcast message asking to the agent subsystem (representing the ontology) for the web services that implement a determined concept. The agents that represent such a concept will respond by returning a list of web service links in the UDDI register. The localizer agent registers the received responses and returns all results. 


\subsubsection{Checking subsystem}

The checking subsystem is responsible for verifying that the web services previously obtained hold the constraints imposed by the user, from the information given by the checking coordinator. This process develops three stages of checking to reach the desired filtering:

1. Input checking: It determines whether the input format of a web service fits the description given by the user.

2. Output checking: It determines whether the output format of a web service fits the description given by the user.

3. Checking of the process: It determines whether the exchange of SOAP messages for the web service is coherent with the description of the user.

\subsection{Composition system}

Once obtained diagram BPMN with the web services associated with each activity in the diagram, the composition of web services through the composition system will be run to achieve the solution web service by using standard WS-BPEL 2.0 [52]. The composition system runs in response to the called from cloud-functionality composeService given in Section 4.1. composeService receives as input an XML document with diagram BPMN and runs the composition system by passing a message to the manager agent.

There are several alternatives to carry out the composition process of web services, such as the variants explained in [53-56]. We have assumed the variant given in [56], which makes a mapping from graphic standard BPMN to language BPEL by extracting the information from the WSDL files corresponding to the web services associated with each activity in the diagram and the input XML document. To define the remaining properties of the WS-BPEL composition, we have used the method developed in [56], which defines the following steps:

1. Definition of the beginning of the process.

2. Definition of the end of the process. 
3. Mapping of the parallel flow.

4. Synchronization of the parallel flow and

5. Mapping of loops.

Summarizing, the process of composing web services to obtain the required solution is carried out by the composition system. It receives the start request from the manager agent, for which the composition coordinator agent creates an agent for each activity (or service), Figure 12. These agents retrieve the needed information from the WSDL files of the associated web services and return such information to the composition coordinator agent, which generates the WS-BPEL by basing on the method given in [56]. Note that this process has an additional improvement since it performs on a distributed environment of cloud computing.

\section{*** Figure 12 here $* * *$}

\subsection{Insertion of new web services to the platform}

The platform on which lies architecture IPCASCI is scalable with respect to the registered $w e b$ services. That is, it is possible inserting and registering new web services in such a way that in a later running, they can be available to use. Since the whole system is included in a cloud environment, there is no a limitation with respect to the size of the service repository and it can grow up indefinitely. Then, to insert a new web service in the platform and can be localized, we must have the following items:

1. A file with description WSDL of the web service;

2. To be registered in the UDDI register;

3. File WSDL must have the ontological annotations corresponding to the semantic content it represents;

4. In case that the references to the semantics annotations do not appear in the ontologies defined in the platform, then a new ontology should be added and running a process to transform its content into computable one. 
To insert the new web service in the platform, the register subsystem given by the search system is responsible for carrying out the operations allowing the localization of such a service through semantics search. Additionally, the semantics annotations inserted into the web service will be obtained from the WSDL file and for each annotation, the agent that implements the ontological concept will be found. The reference to such a web service in the UDDI register will be added to the list of services associated with the agent.

\section{A case study for arquitecture IPCASCI}

In this section we have given an implementation of architecture IPCASCI on a practical case study. The goal of this study is to evaluate our proposal on a real environment and in this way, to develop a practical tool representing the case study and using Algorithm AWSDC given in Section 3. In this sense, a set of parameters of such a tool has been evaluated to achieve results giving us a general overview of the reliability of architecture IPCASCI. Then, we can start by introducing the proposal of the case study in the following way:

This case study develops a tool to build a web service (in automatic way) to reserve a book through the book lending system in a library. The web service must have as input the identifier of the user that carries out the request and the required book. The task of the web service is then to check whether the user is registered in the system and its subscription has not expired. After successfully checking the above, the web service must then verify that if the book is available for loan. Moreover, it would also check whether the user has not exceeded the maximum number of books loaned by the library. If all the above conditions are held, then the loan is carried out. Otherwise, the user will be notified (for example, by e-mail) that the request has been denied along with the reason for it. 
Hereinafter, we are going to show the processes included in the tool to convert the requirements of the user into modules and relationships oriented to the composition of existing web services to build the solution web service.

\subsection{Requirements of the user}

To implement the above case study we have developed a tool called LibraryBookReserve (or LBR for short) that has been implemented on the Java language. This tool couples an assistant for the requirement input of the user in order to process such information to later obtain the solution web service. Thus, the assistant allows the user to introduce the specifications through a graphic interface where possible to define the modules that will integrate the solution web service. Additionally, for every single module, the assistant also provides an interface to introduce its functionality (semantic concepts) and the relationship with other modules. Particularity, a module has been represented by a set of attributes, such as: name, type, action, domain, input, output and interconnection with other modules. In this sense, the modules generated from the case study have been defined in Table 2.

$$
\text { *** Table } 2 \text { here *** }
$$

As explained, the modules have been described by an ontology to define their behavior and actions. Therefore, for each module in the above table has been introduced its semantics as shown in Tables from A1 to A4 (Section A.2 in Appendix A). The attributes of the relationships between these modules have been given in Tables A5-A6 (Section A.2 in Appendix A). Figures A3, A4 (Section A.2 in Appendix A) and a general view in Figure 13 show definition examples of module and relationship from tool LibraryBookReserve. Note that we can build a flow diagram representing the information of the user from the defined modules and relationships. Thus, such a diagram has been generated and stored in an XML file by the tool assistant in order to start the analysis process. 


\subsection{Analysis process of the input requirements}

As previously stated, the input requirements are passed (in XML format) to agent-based virtual organization analysis system. To do this, the tool calls functionality analyze\&Discover (see Section 4.1), which in turn calls the analysis system in order to build the agent subsystem (Section 5.1) representing the domain ontology from the modules and relationships given as input to the functionality. As a result, a diagram BPMN (BPD) is generated by converting the agents into activities. Finally, it is achieved an XML file with the information of diagram BPMN. This diagram will later be completed by the information given from agent-based virtual organization search system (Section 5.2). Thus, functionality analyze\&Discover returns the final result in an XML document.

\subsection{The discovery process}

The discovery process of the web services related to the agents of the agent subsystem (built by the analysis system) is started from search system and has two parts:

1. Finding the web services associated with the semantic concepts for each agent.

2. From the achieved web services, will be filtered those that meet the syntax given by the agents representing the ontological concepts (that is, holding the syntax for input, output and preconditions).

According to this, Table A7 (Section A.2 in Appendix A) shows the web services available in the platform of the case study, which can be used in the web service composition process. Then, once obtained the web services associated with the ontological concepts for every agent, the filtering process is run as a second part. The goal is to extract those services fitting the format given in the description of each agent, that is: by checking input, output and the process each one carries out. Particularly, to check a web service meets the ontological requirements, its file WSDL is also analyzed to verify the list of input and output semantic concepts, preconditions and actions. The web services filtered by the search system have been shown in Table A8 (Section A.2 in Appendix A). With the above filtered process 
is completed the process of analysis and discovery invoked by functionality analyze\&Discover, which returns diagram BPMN and the list of web services associated with each activity.

\subsection{Validation of the solution}

When functionality analyze\&Discover completes its task, the tool has found the web services associated with the modules defined by the user and has built diagram BPMN. Then, to build the solution web service it is necessary:

1. For every activity, selecting the web service to use in diagram BPMN (note that in Table A8, Section A.2 in Appendix A, there is more than one web service for a module).

2. Connecting inputs and outputs for each activity in diagram BPMN.

Hence, the user should select a web service from list associated with each activity in the diagram BPMN by using the graphic assistant of the tool. That is, the graphic assistant of the diagram BPMN has three sections representing the information to process by the tool (see example in Figure 14):

1. Diagram $B P M N$ : This section displays diagram BPMN from the XML file returned by analyze\&Discover. The tool graphic interface allows us selecting and moving the components present in the diagram.

2. List of activities: This section displays the list of activities integrating diagram BPMN (associated with the modules defined by the user). For every activity has been included the following information:
a. Activity name
b. Performed action
c. Domain
d. Input list
e. Output list
f. List of preconditions to meet for the activity performs well. 
3. Found web services: List of web services available for the selected activity. By default, the first web service in the list has been selected by tool, but the user can select any other from the list.

$$
\text { *** Figure } 14 \text { here } * * *
$$

The web services selected for each activity/module of our case study have been shown in Table A9 (Section A.2 in Appendix A). To complete the validation process of the solution proposed by the tool and build the expected web service, the assistant of the diagram BPMN creates the data flow between the activities of such a diagram. That is, each activity in the diagram receives certain inputs that can come from the before activity or the input of the web service to build.

\subsection{Composition of the solution web service}

Completed the association process of web services with the activities of diagram BPMN and stated their connections; the tool can carry out the web service composition by calling functionality composeService (see Section 4.1). This functionality applies standard WSBPEL and the algorithm given in [56] to make the composition process. Basically, composeService invokes the manager agent by passing diagram BPMN. The manager agent processes the information and calls the composition coordinator agent of the composition system. The above agent builds an agent for each web service (or activity) of diagram BPMN. These new agents are responsible for achieving the necessary data from files WSDL (web service description) for the composition process (see Figure 12). Finally, the composition coordinator agent receives all the information from files WSDL to generate file WS-BPEL. This file has been generated by modeling the components in composition BPEL for the solution web service, namely:

1. Flow control;

2. Beginning of the service; 
3. Exchange of massages and

4. Events.

\subsection{Evaluation of the case study}

In this subsection we evaluate tool LibraryBookReserve and the solution web service generated from the composition process. The evaluation has been made taking into account concepts of software engineering to check the internal quality of the composition process. The internal quality of a computer program is related to white-box testing [57], which is the examination of procedural details of the software. It evaluates logical paths in the software by providing specific sets of conditions and/or loops. Moreover, the state of the software on different points of its implementation can be verified in order to determine whether the current state of the software matches the expected state.

For our case study, we have used basis path testing as a white-box testing. This test has been introduced to evaluate the different logical paths of the flow diagram used to build the solution web service through tool LibraryBookReserve. Therefore, it measures the performance of the internal logic structure of the solution web service that has automatically been built by the tool. With this test we want to prove that the automatic process of web service composition performs well.

\subsubsection{Basis path testing for the solution web service (white-box testing)}

Basis path testing is a technique of white-box testing proposed in [58], which allows test case designers to create a measure evaluating the logic complexity of a procedural design and uses it as a guide in the definition of a basis set of running paths. The goal of this test is to determine the number of independent paths for a set of statements (in our case, the flow diagram of the solution web service) to create test cases ensuring the running of each logical path, at least once. This will prove correctness and completeness of the reached solution.

To evaluate the flow diagram of the solution web service as shown in Figure 15, it has been created a test case for every possible path of such a diagram. This figure displays the flow diagram connecting the web services that integrate the service composition (see Tables 
A5, A6 and A9; Section A.2 in Appendix A) to obtain the solution specified by the user. Note that this flow diagram represents the tasks created by the tool to run the solution web service, being these tasks, the web services integrating the service composition. Then, from this diagram, all possible logical paths have been computed by building the associated flow graph given in Figure 16.

\section{*** Figure 15 here ***}

Graph $G$ in Figure 16 shows the flow graph associated with the flow diagram in Figure 15. Thus, this graph represents a logic control structure more suitable than the flow diagram to compute all logical paths [57]. In general terms, nodes in the graph mean one or more procedural statements and numbers identify such statements in the flow diagram. Contiguous statements can be in the same node. Arcs represent the control flow and mean the same as in the flow diagram. Predicate nodes represent conditional statements from the flow diagram and can be identify because two or more arcs emerge from them as shown in Figure 15. In this case, the predicate nodes have been identified in the graph with numbers 2,7 and 8 . Then, from this graph we can compute cyclomatic complexity in order to determine the number of running independent paths of the flow diagram. Cyclomatic complexity is a software metrics providing an upper bound for the number of tests that must be conducted to ensure that all statements have been executed at least once. An independent path is any path in the graph (or flow diagram) that introduces, at least, a new set of statements.

$$
\text { *** Figure } 16 \text { here } * * *
$$

In consequence with the above, cyclomatic complexity $V$ of graph $G$ in Figure 16 can be computed according to [57], from expression $V(G)=A-N+2$, where $A$ is the number of arcs in the graph and $N$ is the number of nodes. Then, $V(G)=11 \operatorname{arcs}-8$ nodes $+2=5$. Hence, we can now determine the basis set of linearly independent paths (which has 5 
paths). Table 3 lists the five linearly independent paths from the flow graph, for which we have prepared a test case for each.

\section{*** Table 3 here ***}

For each path in Table 3, a test case has been prepared in order to force the solution web service to run it. As a result, the test cases have proven that each path has successfully been run at least once and the results reached in each case match the expected ones. Therefore, the logic implanted by the flow diagram in Figure 15 (which is based on the user requirements) and the selected web services have performed well. Additionally, all the previous process of white-box testing has also been applied to each flow diagram different from the one given in Figure 15, for which the same results have been obtained. Note that in the process of web service composition (diagram BPMN); the tool allows us to choose a web service different from the current one, which generates a new flow diagram to check.

\subsection{Results}

In this section we have introduced a case study to present an implementation of our proposal, architecture IPCASCI, which states a model to automatically compose business process. As a first result of the case study, we have given an implementation of IPCASCI as tool LibraryBookReserve, which creates new web services from the composition of web services existing in the platform to reserve books in a library. This result proves the reliability of our proposal, architecture IPCASCI, on a real environment of cloud computing. On the other hand, we have also introduced a validation process of the case study by evaluating the internal logical structure that the solution web service has to run (white-box testing). Since in our case, white-box testing focuses on the control structure of the solution web service; we have also proven the completeness and correctness of tasks performed by the flow diagram run from the process of web service composition (diagram BPMN, Figure 15) to obtain the expected solution. In this sense, the solution web service generated by the tool fits the requirements introduced by the user. Finally, all these results also prove the 
goodness and reliability of the logic structure of the multi-agent system coupled to IPCASCI, which is aimed at the discovery and refinement of web services taking place in the services composition process.

\section{Conclusions}

This paper has proposed an architecture called IPCASCI for agile and automatic composition of business processes on a cloud computing environment. This proposal is able to fit the technological environment, being accessible and useful for the business world. For this approach, the software components to reuse in the composition process have been web services. In this sense, the main characteristic of our proposal is that from a set of specifications (in text format) given by an inexperienced user to build a business process; IPCASCI is able to develop the product as a web service from the ones existing in the platform. To do this, IPCASCI has provided a web service repository in a cloud computing environment; an ontology system in order to represent the necessary knowledge to endow semantics (WSDL-S, Web Service Semantics Language) to the existing web services, and so later facilitate their discovery process and composition; a multi-agent system coupling agentbased virtual organizations aimed at discovering and composing the web services fitting the requirements initially given by the user.

To prove the performance of this proposal, we have presented an implementation of IPCASCI as a tool from a real case study. Therefore, our first result of the case study has been to develop tool LibraryBookReserve to automatically build web services from specifications of the user to reserve books in a library. According to the requirements of the user, this tool provides a graphic interface to introduce such specifications in a simple, intuitive and agile way. In this context, we have also proven through a white-box testing (software metrics), that the internal logic structure of the solution web service (in this case, its flow diagram) built by LibraryBookReserve performs well on the selected application domain. Moreover, by simple modifications of the tool, we can add other application domains, extending its use and so providing reusability. Consequently, this proves that 
architecture IPCASCI is not only useful for the presented case study; it is also useful for any other application domain. Hence, our proposal adapts to the current needs of software development, for which it is possible to create new products in very short time, at a reduced cost and without going through an arduous and external development process.

Finally, keep in mind that our approach has been benefited from the potential of a cloud environment, which introduces a change in the way of exploiting and marketing the company's products [59]. Hence, IPCASCI deals with the following advantages present in systems based on cloud computing: (i) Resources in a cloud environment can be allocated and dissallocated according to needs at any given time; (ii) Resources can be shared or/and allocated to each customer depending on their needs; (iii) Services stored in the cloud are generally based on web technology, a fact that makes them accessible to a broad range of devices with Internet connection; (iv) Once cloud service infrastructure is outsourced, the service provider transfers its business risks (as for example, hardware failures) to the infrastructure providers that are usually more experienced and better equipped to handle such risks.

\section{Author contributions}

JAGC designed and implemented the proposed architecture supervised by JMC. JAGC and JAC-G wrote the paper. JMC and JAC-G provided the comments and the discussion. All authors read and approved the final manuscript.

\section{Competing interests}

The authors declare that they have no competing interests.

\section{Appendix A. Information supporting architecture IPCASCI}

\section{A.1. Example of converting the agent subsystem into a BPD diagram}


This example shows the transformation of the modules and their relationships defined by the user into an agent subsystem and later into the BPD diagram. Then, in this case we have defined seven modules, from Module1 to Module7, where modules 1 and 7 are the ones of startup and end respectively. The following relationships between the introduced modules have been defined:

1. Modulo1 Parallel output to Modulo2, Modulo3

2. Modulo2 If [Condition] Modulo4 Else Modulo5

3. Modulo6 Follows Modulo4

4. Modulo6 Follows Modulo5

5. Modulo7 Follows Modulo6

6. Modulo7 Follows Modulo3

The agent subsystem created by the analysis system corresponding to the above input has been shown in Figure A1. This agent subsystem has been mapped to standard BPMN in Figure A2 by applying the transformations given in Table 1, Section 5.1.

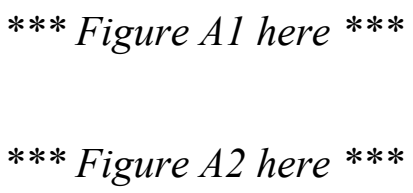

A.2. Complementary information of the case study

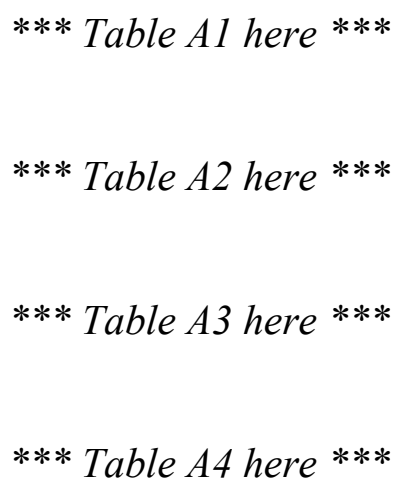




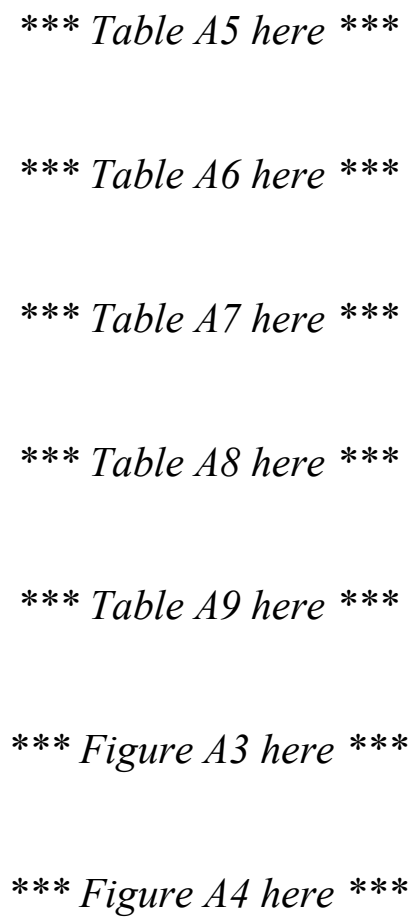

\section{References}

[1] K. Schmid, Top Productivity through Software Reuse, 12th International Conference on Software Reuse, Lecture Notes in Computer Science, ICSR, Springer 6727, 2011.

[2] J. S. Poulin, Measuring software reuse. Principles, practices, and economic models, Addison-WesleyLongman, ISBN-978-0-201-63413-6, 1997.

[3] J. S. Poulin, The Business Case for Software Reuse: Reuse Metrics, Economic Models, Organizational Issues, and Case Studies, Lecture Notes in Computer Science, Springer 4039 (2006) 439.

[4] M. Lemley and D. O'Brien, Encouraging Software Reuse, Stanford Law Review 49 (1997) 255.

[5] R. D. Shang, K. Mohan, K. R. Lang, R. Vragov, A market mechanism for software component reuse: opportunities and barriers, Proceedings of the 14th Annual International Conference on Electronic Commerce (ICEC '12), ACM (2012) 62-69.

[6] H. Rehesaar, Capability Assessment for Introducing Component Reuse, Lecture Notes in Computer Science, Springer-Verlag 6727 (2011) 87-101.

[7] Y. Xu, N. Singh, S. Deshpande, Reuse by Placement: A Paradigm for Cross-Domain Software Reuse with High Level of Granularity, Lecture Notes in Computer Science, Springer 6727 (2011) 69-77. 
[8] V. C. Garcia, D. Lucrédio, A. Alvaro, E. S. de Almeida, R. P. de Mattos, S. R. de Lemos, Towards a maturity model for a reuse incremental adoption, In: The 1st Brazilian Symposium on Software Components, Architecture and Reuse, Campinas, Sâo Paulo, Brazil (2007) 61-74.

[9] L. Osiecki, M. Phillips, J. Scibilia, Understanding and Leveraging a Supplier's CMMI Efforts: A Guidebook for Acquirers, Software Engineering Institute, Carnegie Mellon University, Pittsburgh, Pennsylvania, Technical Report CMU/SEI-2011-TR-023, 2011

[10] A.E. Walsh, UDDI, SOAP, and WSDL: The Web Services Specification, Pearson Education, 1st edition, 2002.

[11] T. Erl, SOA Design Patterns, The Prentice Hall Service-Oriented Computing Series, 2009.

[12] J. Ralyté, I. Mirbel, R. Deneckère, Engineering Methods in the Service-Oriented Context, 4th IFIP WG 8.1 Working conference on method engineering, 2011.

[13] K. Alizadeh, M. Seyyedi, M. Mohsenzadeh, A Service Identification Method based on Enterprise Ontology in Service Oriented Architecture, International Journal of Information Processing and Management (IJIPM) 3 (2012).

[14] M. Papazoglou, Web Services: Principles and Technology, Pearson Education, Prentice Hall, 2008.

[15] N. Antonopoulos, A. Anjum and L. Gillam, Intelligent techniques and architectures for autonomic clouds, Journal of Cloud Computing: Advances, Systems and Applications (2012) 1-12.

[16] A. Stage and T. Setzer, Network-aware migration control and scheduling of differentiated virtual machine workloads, In: Proceedings of the ICSE Workshop on Software Engineering Challenges of Cloud Computing (CLOUD '09), IEEE Computer Society, Washington, DC, USA (2009) 9-14.

[17] T. Duy, Y. Sato, Y. Inoguchi, A prediction-based green scheduler for datacenters in clouds, IEICE Trans Inf Syst E94-D 9 (2011) 1731-1741.

[18] Cloud Computing, Retrieved at June 03, [http:/ / fclose.com/ b/ cloud-computing/ article/ mrcc-adistributed-c-compiler-syste m-on-mapreduce/], 2011.

[19] EuroCloud Deutschland_eco eV, Eurocloud star audit saas certificate, http://www.saas-audit.de, 2011.

[20] P. T. Jaeger, J. Lin, J. M.Grimes, S. N. Simmons, Where is cloud? Geography, Economics, Environment, and Jurisdiction in Cloud Computing. ISSN-1396-0466, 4 (2009).

[21] F. Dölitzscher, C. Reich, A. Sulistio, Designing Cloud Services Adhering to Government Privacy Laws, In: Proceedings of 10th IEEE International Conference on Computer and Information Technology (CIT), Furtwangen, Germany (2010) 930-935.

[22] P. Massonet, S. Naqvi, C. Ponsard, J. Latanicki, B. Rochwerger, M. Villari, A Monitoring and Audit Logging Architecture for Data Location Compliance in Federated Cloud Infrastructures, In Parallel and Distributed Processing Workshops and Phd Forum (IPDPSW), IEEE International Symposium on (2011) 1510-1517. 
[23] M. Schaaf, A. Koschel, S .G. Grivas, I. Astrova, An active DBMS style activity service for cloud environments. Cloud Computing: The First International Conference on Cloud Computing, GRIDs, and Virtualization in Cloud Computing, ComputationWorld IARIA, ISBN: 978-1-61208-106-9 (2010) 80-85.

[24] A. Sulistio, C. Reich and F. Dölitzscher, Cloud Infrastructure \& Applications - CloudIA, In: Proceedings of the 1st International Conference on Cloud Computing (CloudCom), Beijing, China (2009) 583-588.

[25] S. Rodríguez, Y. de Paz, J. Bajo and J. M. Corchado, Social-based Planning Model for Multiagent Systems, Expert Systems with Applications 38 (2011) 13005-13023.

[26] V. Dignum, J. Vazquez-Salceda, F. Dignum, OMNI: Introducing Social Structure, Norms and Ontologies into Agent Organizations. Proceeding of 3rd International Workshop on Programming Multi-Agent Systems, Utretch, The Netherlands (2005) 181-198.

[27] M. Escriva, J. Palanca, G. Aranda, A. García, V. Julian, V. Botti, A Jabber-Based Multi-Agent System Platform, Proceeding of 5th International Joint Conference on Autonomous Agents and Multiagent Systems, Hakodate, Japan (2006) 1282-1284.

[28] A. Maedche and S. Staab, Learning Ontologies for the Semantic Web, In Semantic Web Workshop, Hongkong, China, 2001.

[29] B. Chandrasekaran and J. R. Josephson, What Are Ontologies, and Why Do We Need Them?, Ohio State University V. Richard Benjamins, University of Amsterdam, 1999.

[30] N. Noy and D. McGuinness, Ontology development 101: A guide to creating your first ontology, Technical Report SMI-2001-0880, Stanford Medical Informatics (SMI), Department of Medicine, Stanford University School of Medicine, 2001.

[31] N. Guarino, Formal Ontology in Information Systems, Proceedings of FOIS'98, Trento, Italy, 6-8 June, Amsterdam, IOS Press, 1998.

[32] F. López, Overview of Methodologies for Building Ontologies, In Proceedings of IJCAI-99 workshop on Ontologies and Problem-Solving Methods (KRR5) in Stockholm Sweden, August 2, 1999.

[33] C. Pedrinaci, C. Brelage, T. Van Lessen, J. Domingue, D. Karastoyanova, F. Leymann, Semantic business process management: scaling up the management of business processes, In: 2nd IEEE International Conference on Semantic Computing (ICSC), 4-7 Aug 2008, Santa Clara, CA, USA, 2008.

[34] Z. Su, H. Chen, L. Zhu, Y. Zeng, Framework of Semantic Web Service Discovery Based on Fuzzy Logic and Multi-phase Matching, Journal of Information \& Computational Science 9:1 (2012) 203-214.

[35] K. Sycara, M. Paolucci, A. Ankolekar, N. Srinivasan, Automated discovery, interaction and composition of Semantic Web services. Web Semantics: Science, Services and Agents on the World Wide Web, Elsevier 1 (2003) 27-46.

[36] J. M. García, D. Ruiz, A. Ruiz-Cortés, Improving Semantic Web Services Discovery Using SPARQLBased Repository Filtering, Web Semantics: Science, Services and Agents on the World Wide Web, Elsevier 17 (2012) 12-24. 
[37] M. Owen and J. Raj, BPMN and Business Process Management. Introduction to the New Business Process Modeling Standard, Popkin Software, www.popkin.com, 2003.

[38] J. Pasley, How BPEL and SOA Are Changing Web Services Development, Published by the IEEE Computer Society, IEEE Internet Computing (2005) 60-67.

[39] M. Schaaf, A. Koschel, S. G. Grivas, I. Astrova, An active DBMS style activity service for cloud environments. Cloud Computing: The First International Conference on Cloud Computing, GRIDs, and Virtualization in Cloud Computing, in ComputationWorld IARIA (2010) 80-85.

[40] S. Rodríguez, D. Tapia, E. Sanz, C. Zato, F. de la Prieta, O. Gil, Cloud Computing Integrated into ServiceOriented Multi-Agent Architecture, Balanced Automation Systems for Future Manufacturing Networks, IFIP Advances in Information and Communication Technology, Springer 322 (2010) 251-259.

[41] B. Cao, B. Li, Q. Xia, A Service-Oriented Qos-Assured and Multi-Agent Cloud Computing Architecture, Cloud Computing, Lecture Notes in Computer Science, Springer-Verlag Berlin Heidelberg 5931 (2009) 644-649.

[42] J. Bajo, C. Zato, F. de la Prieta, A. de Luis, D. Tapia, Cloud Computing in Bioinformatics, Distrib. Computing \& Artif. Intell., AISC, Springer-Verlag Berlin Heidelberg 79 (2010) 147-155.

[43] IBM, RESTful Web services: The basics, http://www.ibm.com/developerworks/webservices/library/wsrestful/, 2008.

[44] E. Newcomer. Understanding Web Services: XML, WSDL, SOAP, and UDDI (independent Technology Guides). Addison Wesley, 2002.

[45] E. Cerami. Web services Essentials - Distributed Applications with XML-RPC, SOAP, UDDI \& WSDL. O’RELLY, 2002.

[46] F. M. T. Brazier, V. Dignum, M. N. Huhns, C. Derksen, F. Dignum, T Lessner, J. A. Padget, T. B. Quillinan, M. P. Singh, Agent-based organisational governance of services, Multiagent and Grid Systems 8 (2012) 3-18

[47] D. Tapia, S. Rodríguez, J. Bajo and J. M. Corchado. FUSION@, A SOA-Based Multi-agent Architecture. International Symposium on Distributed Computing and Artificial Intelligence (DCAI), Springer-Verlag Berlin Heidelberg 50 (2009) 99-107.

[48] J. Hendler, T. Berners-Lee, E. Miller, Integrating Applications on the Semantic Web, Journal of the Institute of Electrical Engineers of Japan 10 (2002) 676-680.

[49] M. d'Aquin and N. Noy, To publish and find ontologies? A survey of ontology libraries, Web Semantics: Science, Services and Agents on the World Wide Web, Elsevier 11 (2012) 96-111.

[50] D. Martin, M. Burstein, J. Hobbs, O. Lassila, D. McDermott, S. McIlraith, S. Narayanan, M. Paolucci, B. Parsia, T. Payne, E. Sirin, N. Srinivasan, K. Sycara, OWL-S: Semantic Markup for Web Services, W3C Member Submission, http://www.w3.org/Submission/OWL-S, 2004. 
[51] J. Miller, K. Verma, P. Rajasekaran, A. Sheth, R. Aggarwal, K. Sivashanmugam, WSDL-S: Adding Semantics to WSDL, LSDIS Lab, University of Georgia, http://1sdis.cs.uga.edu/projects/meteor-s/, 2004.

[52] R. Akkiraju, J. Farrell, J. Miller, M. Nagarajan, M. Schmidt, A. Sheth, K. Verma, Web Service Semantics - WSDL-S, W3C Member Submission, http://www.w3.org/Submission/WSDL-S/, 2005.

[52] A. Arkin, S. Askary, B. Bloch, F. Curbera, Y. Goland, N. Kartha, K. Liu, S. Thatte, P. Yendluri and A. Yiu, Web Services Business Process Execution Language, Version 2.0 OASIS, 2004.

[53] C. Ouyang, W. van der Aalst, M. Dumas, A. Hofstede, Translating BPMN to BPEL, Digital Repository, Queensland University of Technology, Australia, 2006.

[54] R. Jan and M. Jan, On the Translation between BPMN and BPEL: Conceptual Mismatch between Process Modeling Languages, In Latour, Thibaud \& Petit, Michael (Eds.), The 18th International Conference on Advanced Information Systems Engineering, Proceedings of Workshops and Doctoral Consortium, Namur University, 2006

[55] C. Ouvans, M. Dumas, A. Hofstede, W. van der Aalst, From BPMN Process Models to BPEL Web Services, ICWS '06 Proceedings of the IEEE International Conference on Web Services, IEEE Computer Society Washington, DC, USA (2006) 285-292.

[56] S. White, Using BPMN to Model a BPEL Process, BPTrends, http://www.bptrends.com, 2005.

[57] R. Pressman, Software Engineering: A Practitioner's Approach, Seventh Edition, Ph.D., McGraw-Hill Companies, 2005.

[58] T. McCabe, A Software Complexity Measure, IEEE Trans. Software Engineering, SE-2 (1976) 308-320.

[59] V. Chang, D. Bacigalupo, G. Wills, D. De-Roure, A Categorisation of Cloud Computing Business Models, 10th IEEE/ACM International Symposium on Cluster, Cloud and Grid Computing (2010) 509512. 


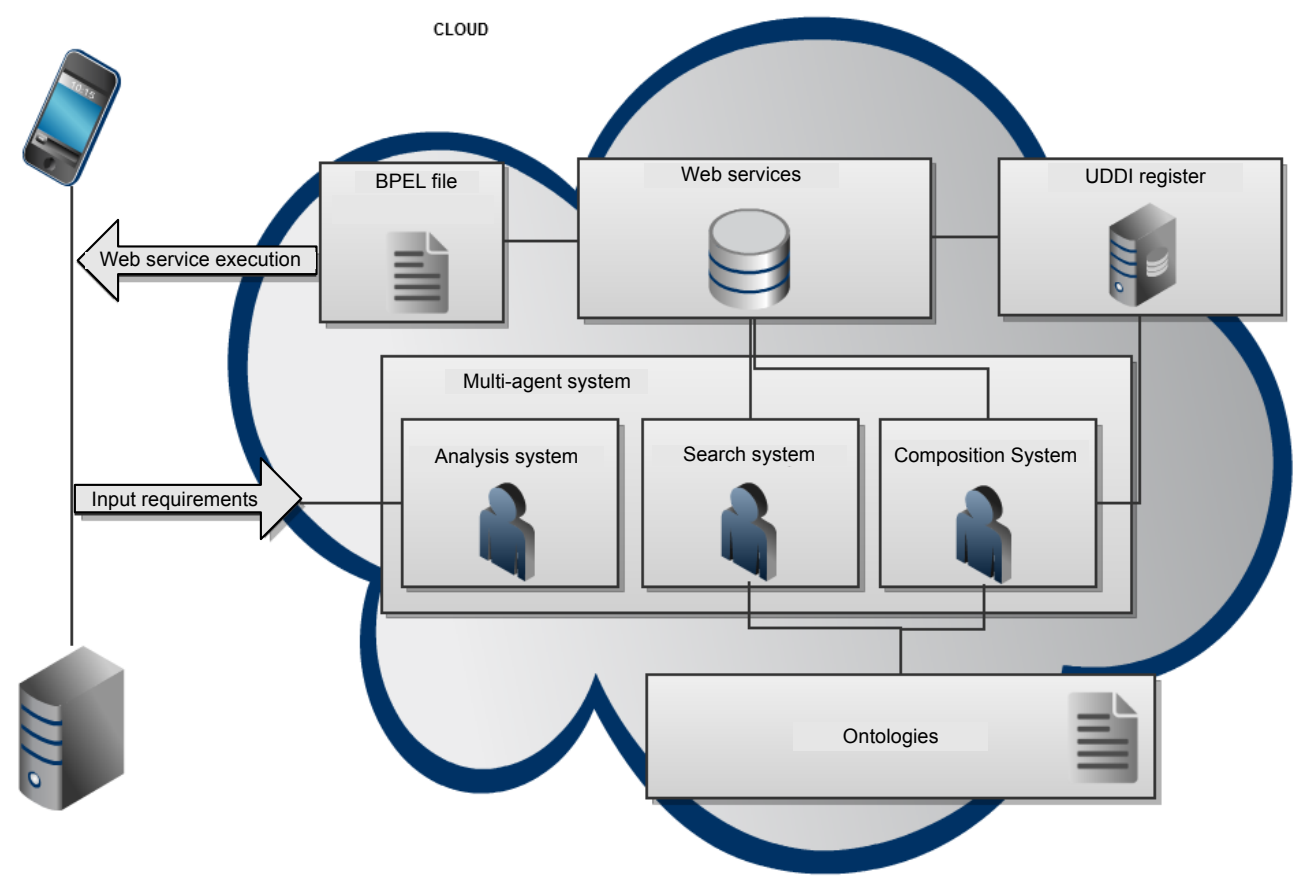

Figure 1: Description of architecture IPCASCI for composition of bussiness processes (web services).

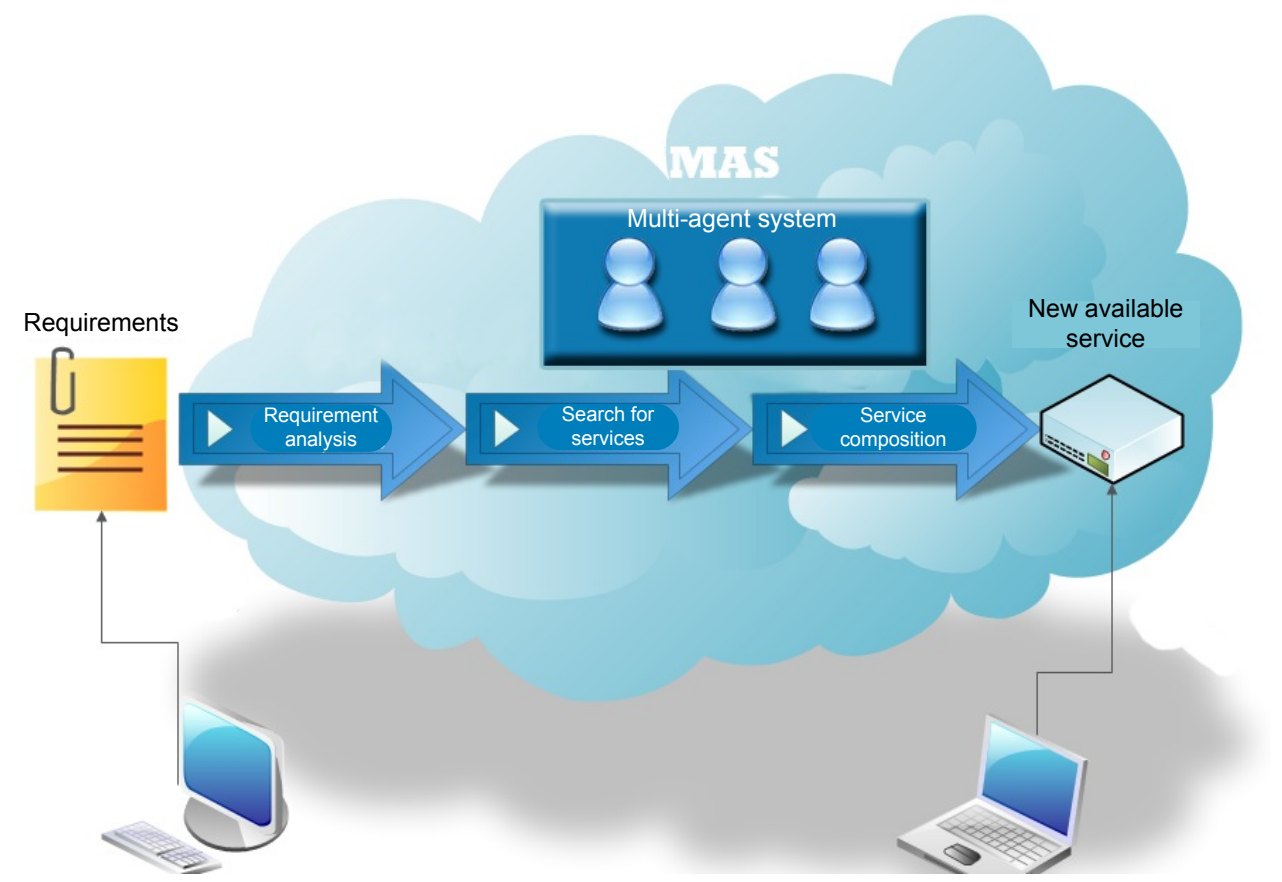

Figure 2: Stages of architecture IPCASCI to obtain the solution web service from the discovery and composition process of web services. 


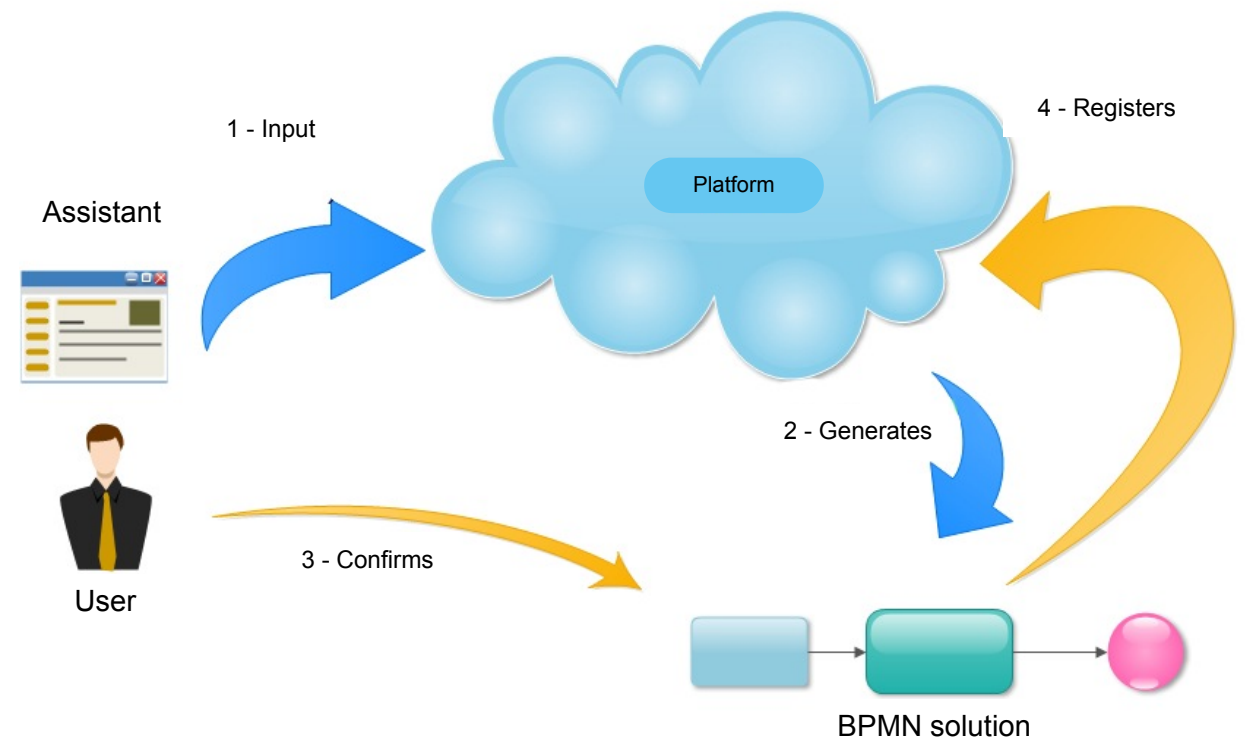

Figure 3: Alternative view (Figure 2) of the process of obtaining the solution web service by architecture IPCASCI.

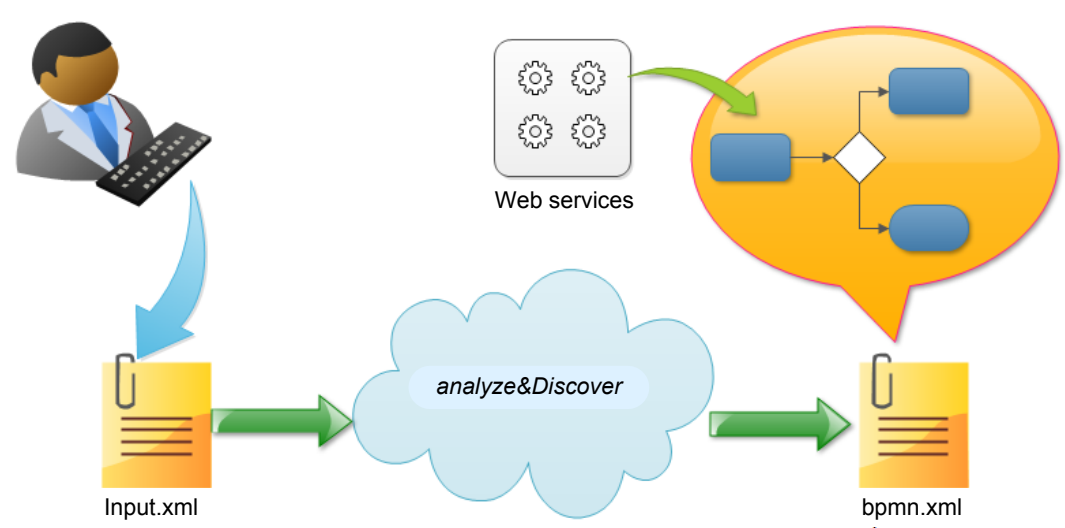

Figure 4: analyze\&Discover cloud functionality interacting with components of architecture IPCASCI. 


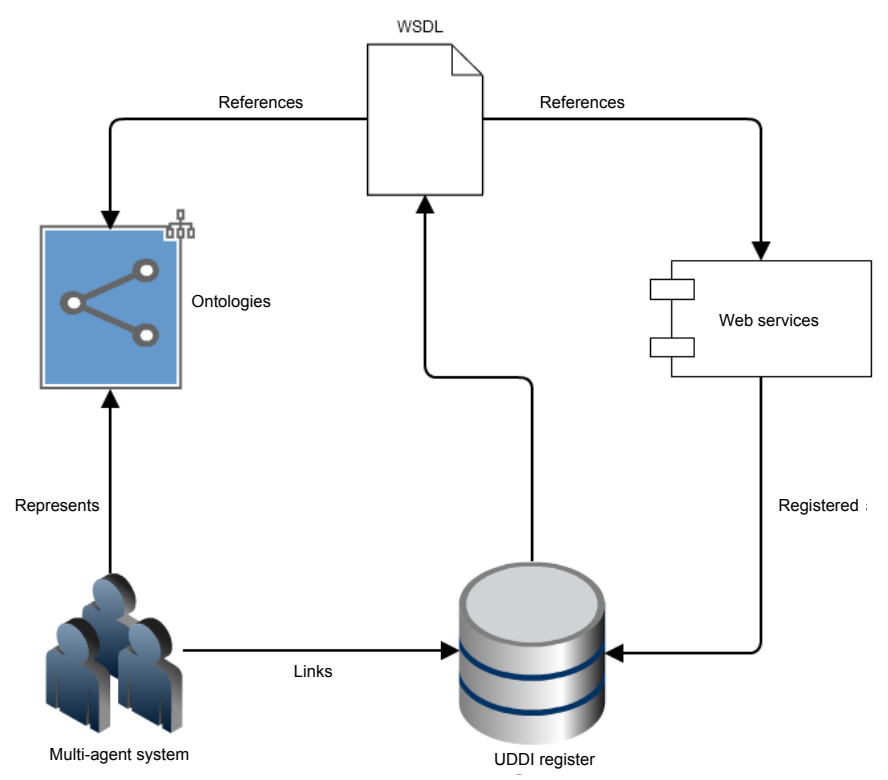

Figure 5: Integration diagram of web services and SOA.

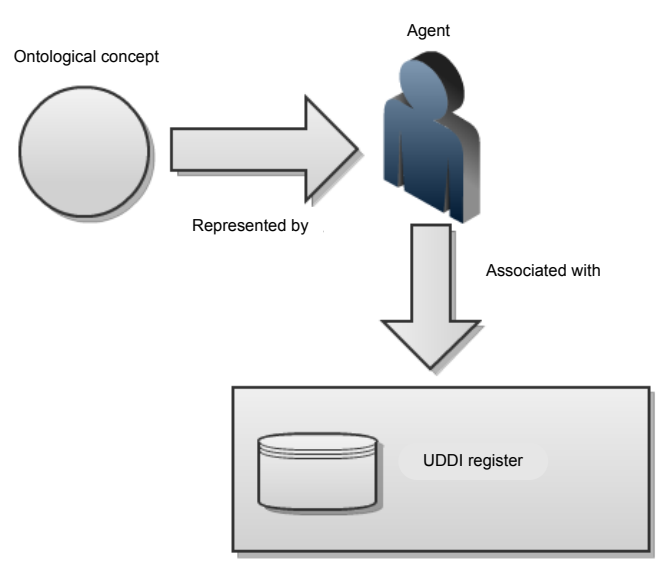

Figure 6: Connecting semantic concepts with entries in register UDDI (web services) through agents. 


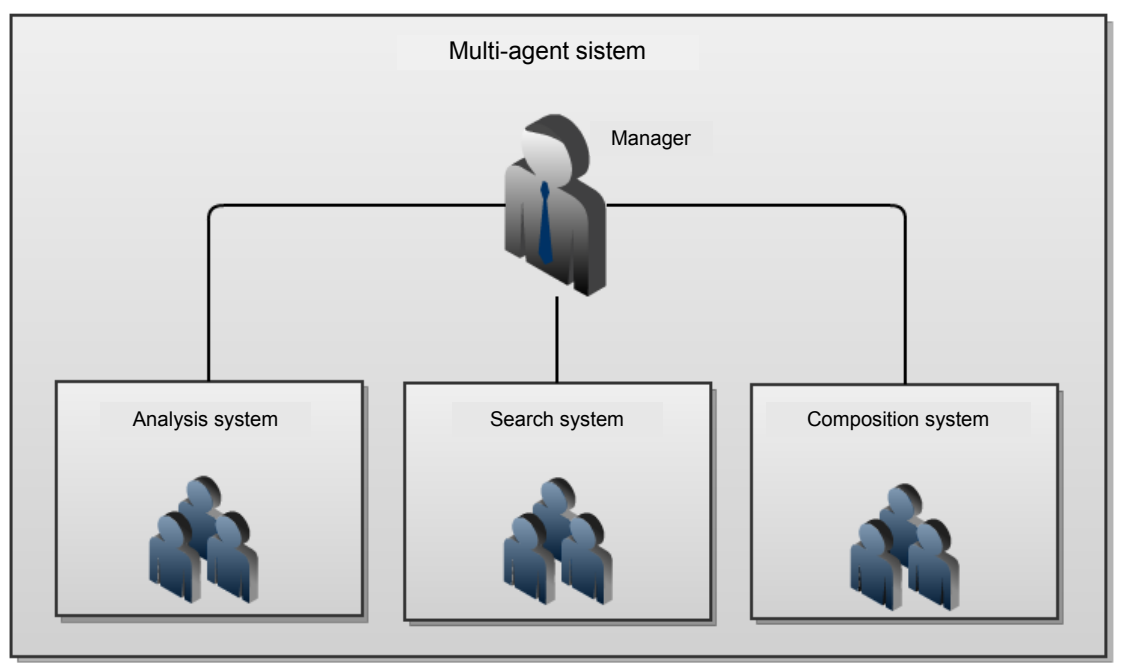

Figure 7: Structure of the multi-agent system (MAS) to represent the domain ontology and manage the requirement input, search and composition of web services.

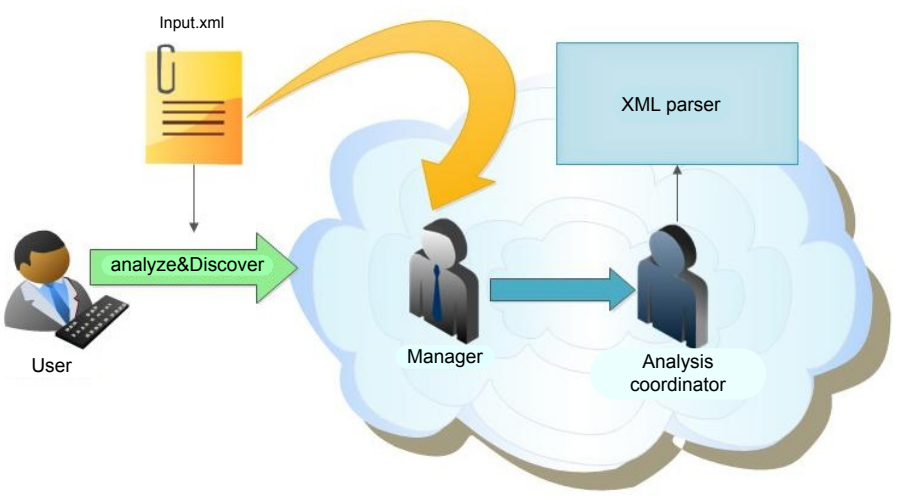

Figure 8: Agent-based virtual organization of the analysis system to analyze and discover the web services associated with the requirements of the user. 


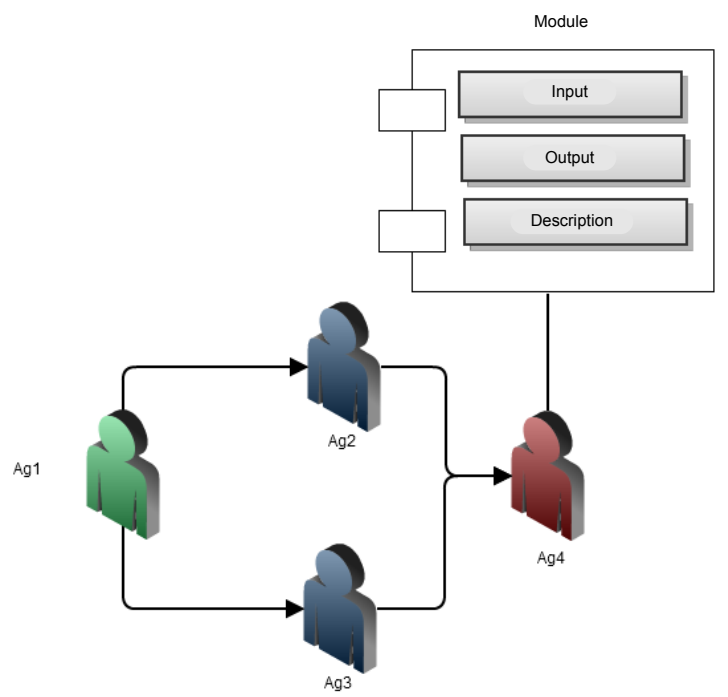

Figure 9: Relationship module-agent and the agent-based graph associated with associated with the modules defined by the user.

Table 1: Relationships between items in the BPMN diagram and items in the agent subsystem.

\begin{tabular}{ll}
\hline BPMN diagram & Agent subsystem \\
\hline Activity & Agent \\
XOR gate ("X") & [Module_i] If [Condition] then [Module_j] or \\
& [Module_i] If [Condition] then [Module_j] else [Module_k] \\
AND gate ("“+") & [Module_i] Parallel output to [Module_j], [Module_k] \\
startup and end events & agents marked as startup and end process \\
any other connector & [Module_i] Follows [Module_j]
\end{tabular}




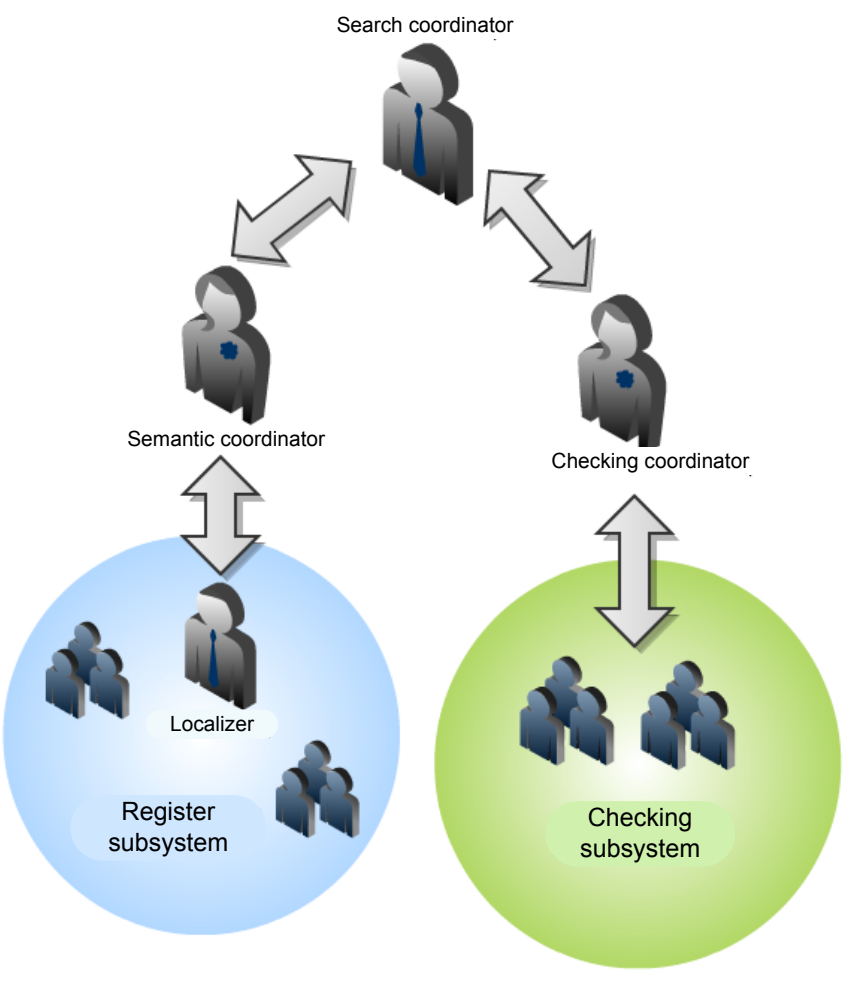

Figure 10: Agent-based virtual organization representing the structure of the search system. 


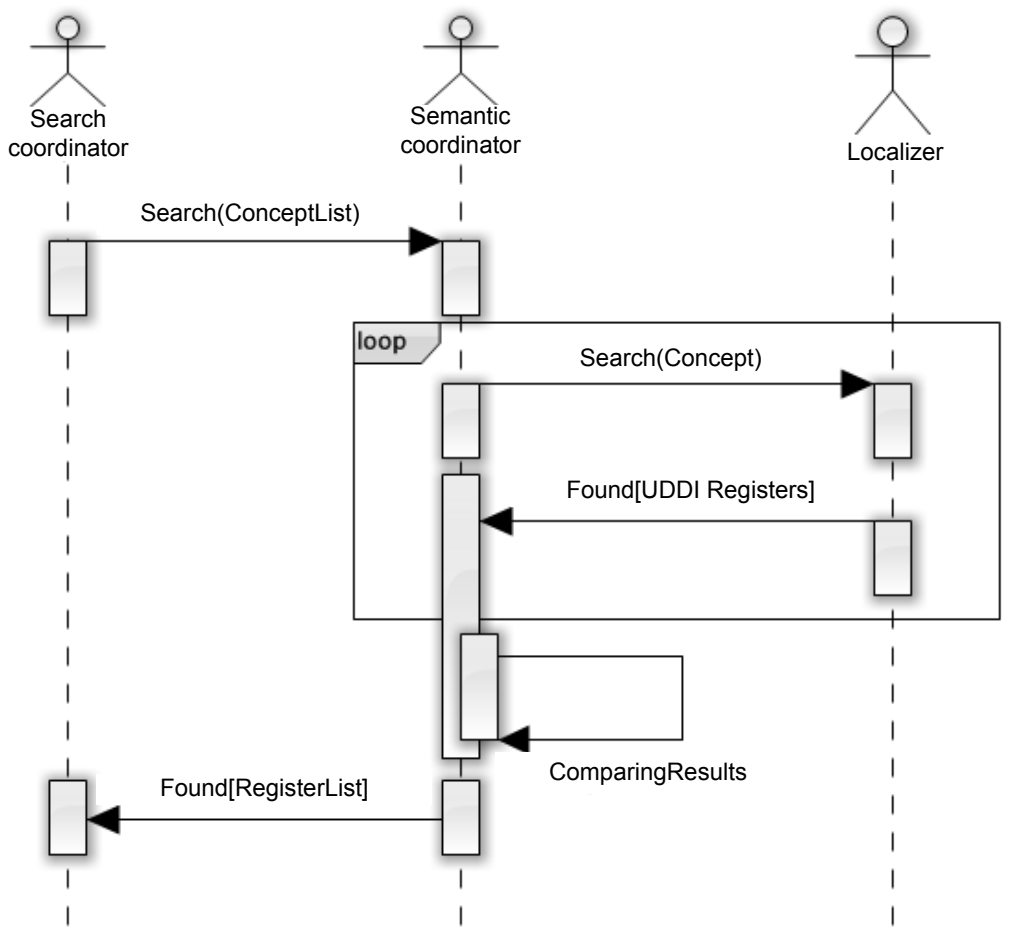

Figure 11: Process of semantics search for the web services associated with a list of ontological concepts.

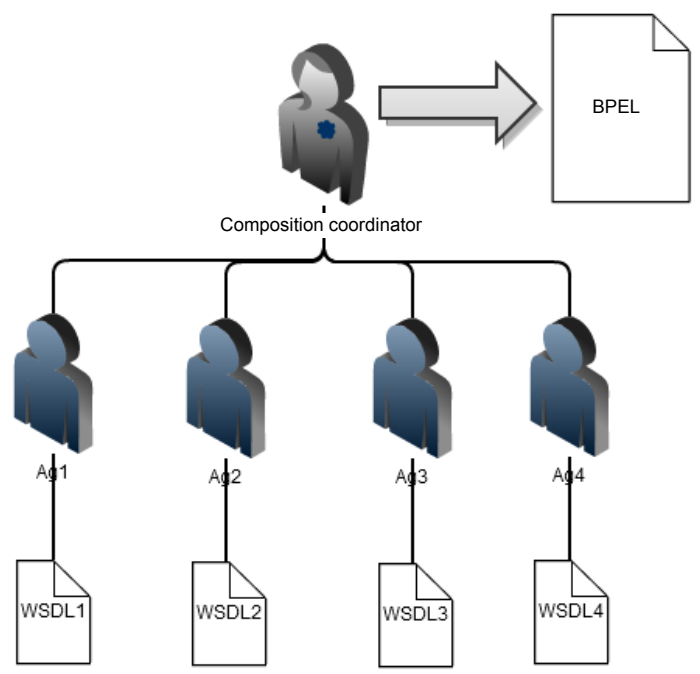

Figure 12: Agent-based composition system to obtain the solution web service. 
Table 2: Definition and meaning of the modules to build the solution web service.

\begin{tabular}{ll}
\hline Module & Description \\
\hline CheckingRecord & $\begin{array}{l}\text { Determine whether the user is registered in the system. } \\
\text { NotifyState }\end{array}$ \\
$\begin{array}{l}\text { Notify to the user that the connection has not been } \\
\text { established and the reason. }\end{array}$ \\
CheckingBook & $\begin{array}{l}\text { Verify the existence of the requested book. } \\
\text { ChekingLoan }\end{array}$ \\
NotifynoStock & $\begin{array}{l}\text { Notify to the user that there is no existence of the requested } \\
\text { book. }\end{array}$ \\
NotifyLoanExceed & $\begin{array}{l}\text { Notify to the user that the maximum number of loans has } \\
\text { been exceeded. }\end{array}$ \\
MakeLoan & $\begin{array}{l}\text { Carry out and register the book loan. } \\
\text { Auxiliary module useful for the later construction of } \\
\text { diagram BPD of graphic standard BPMN. This module is } \\
\text { defined by the system. }\end{array}$ \\
\hline
\end{tabular}

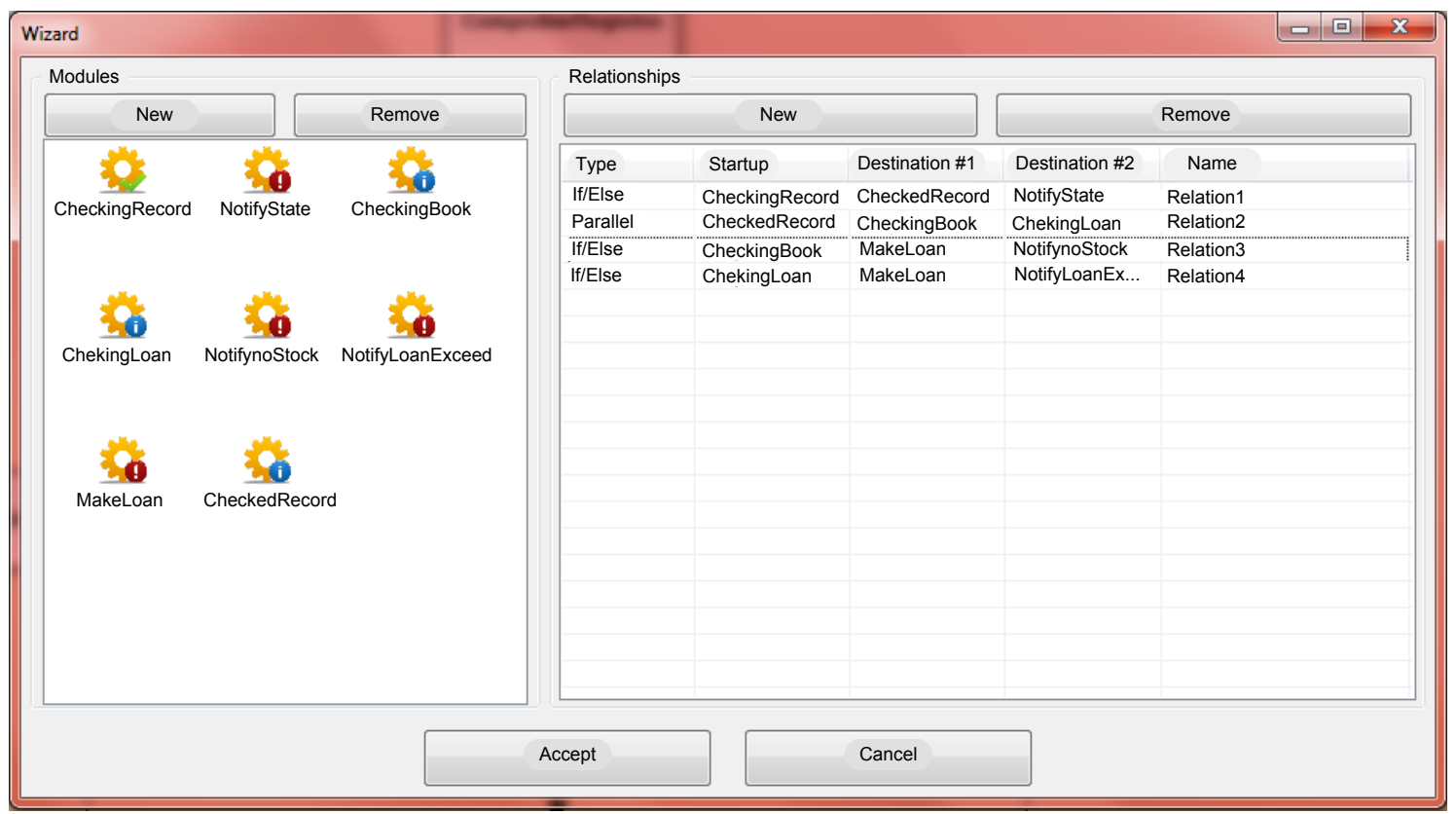

Figure 13: View of tool LibraryBookReserve showing on the left side, the modules defined by the user and on the right, the relationships defined between them. 


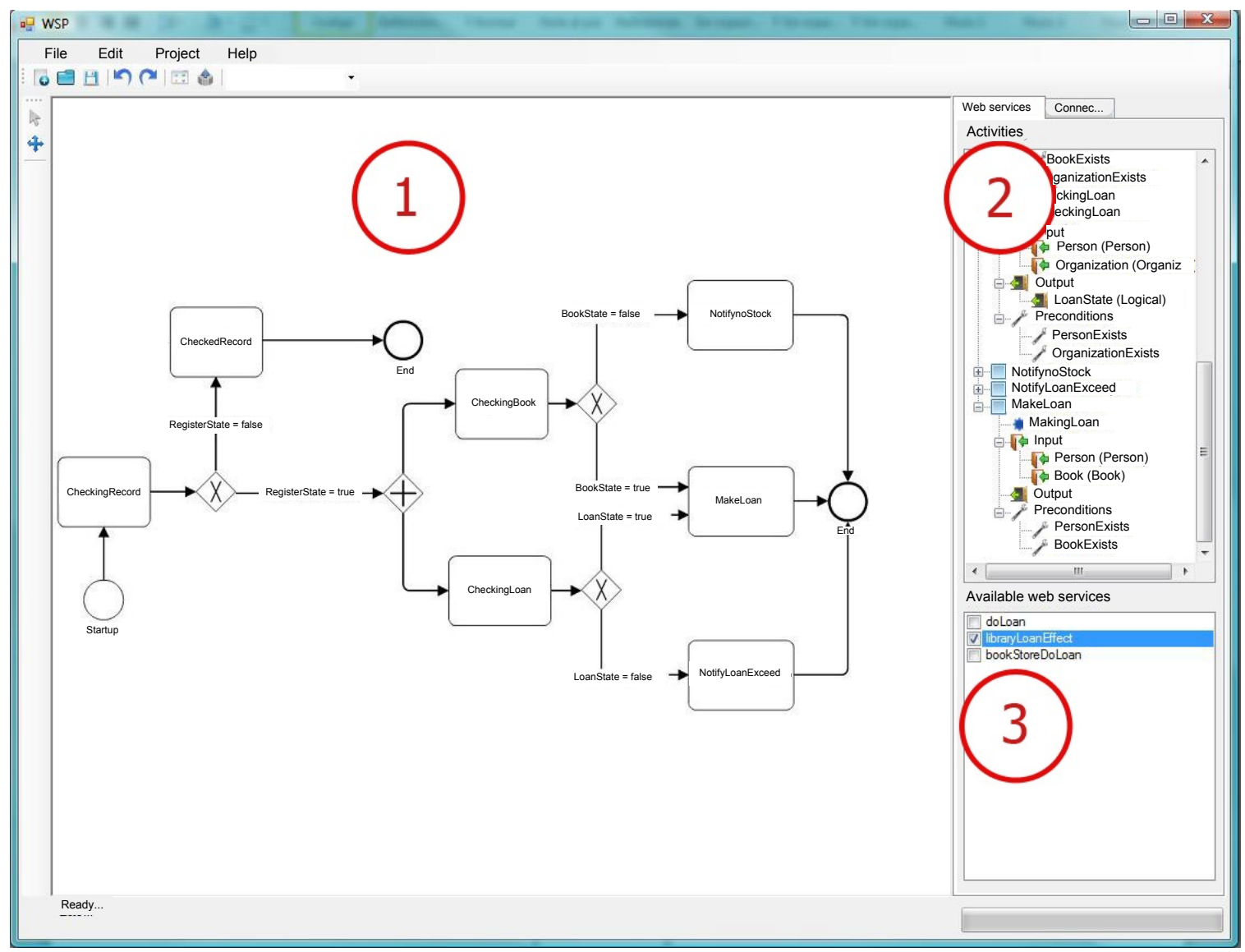

Figure 14: View of tool LibraryBookReserve showing an example where on Part 1 is diagram BPMN of the case study, on Part 2, the list of activities and on Part 3, the available web services. 


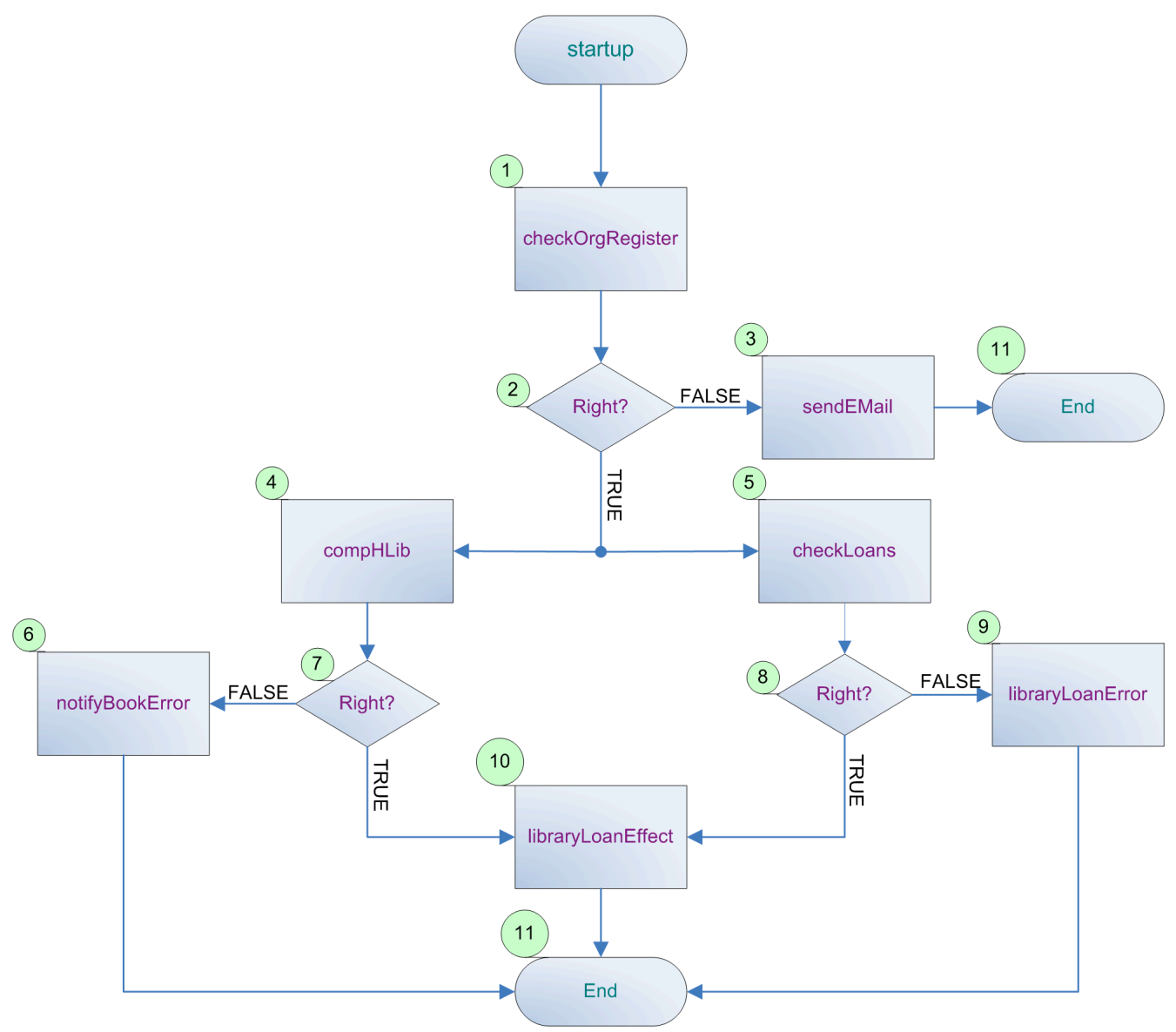

Figure 15: Flow diagram of the web service composition to obtain the solution web service. Rectangles represent the web services selected for the composition. 


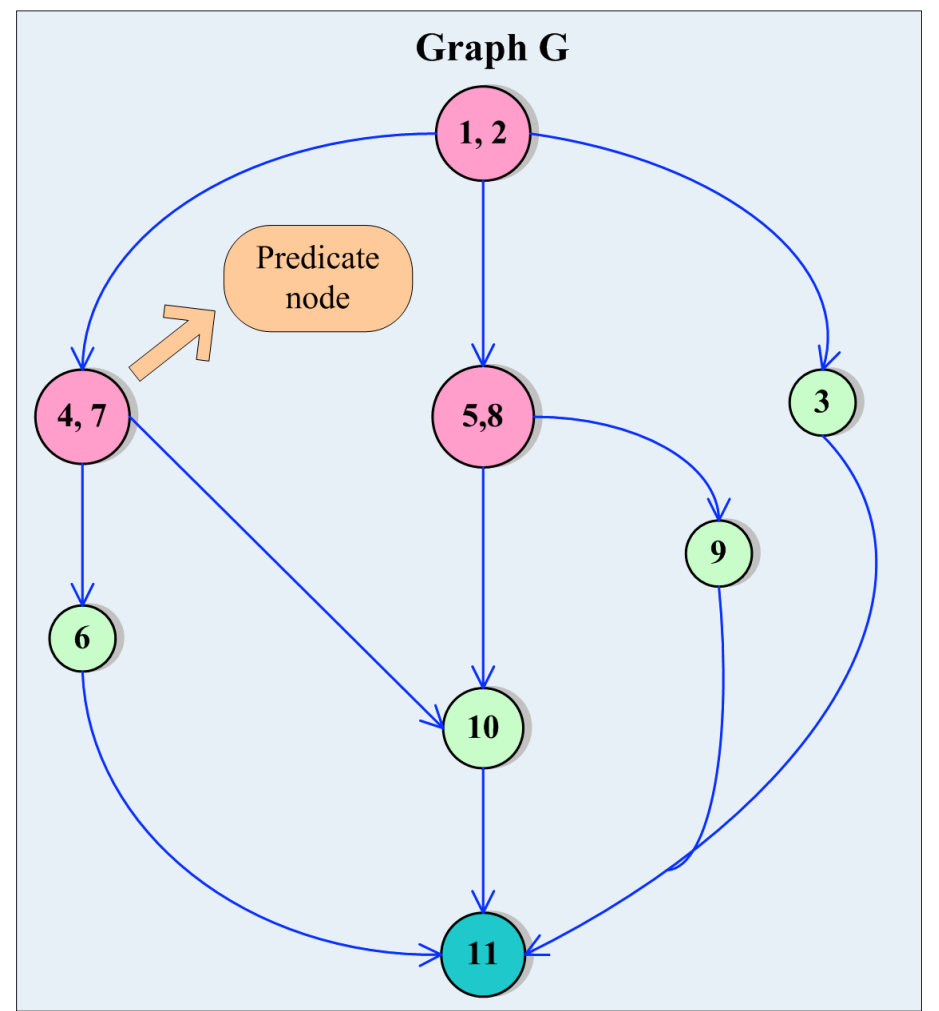

Figure 16: Flow graph associated with the flow diagram in Figure 15. Nodes and numbers represent tasks in the flow diagram.

Table 3: Linearly independent logic paths found in the flow graph in Figure 16.

\begin{tabular}{lc}
\hline & Logical paths \\
\hline Path \#1: & $1-2-4-7-6-11$ \\
Path \#2: & $1-2-4-7-10-11$ \\
Path \#3: & $1-2-5-8-10-11$ \\
Path \#4: & $1-2-5-8-9-11$ \\
Path \#5: & $1-2-3-11$ \\
\hline
\end{tabular}




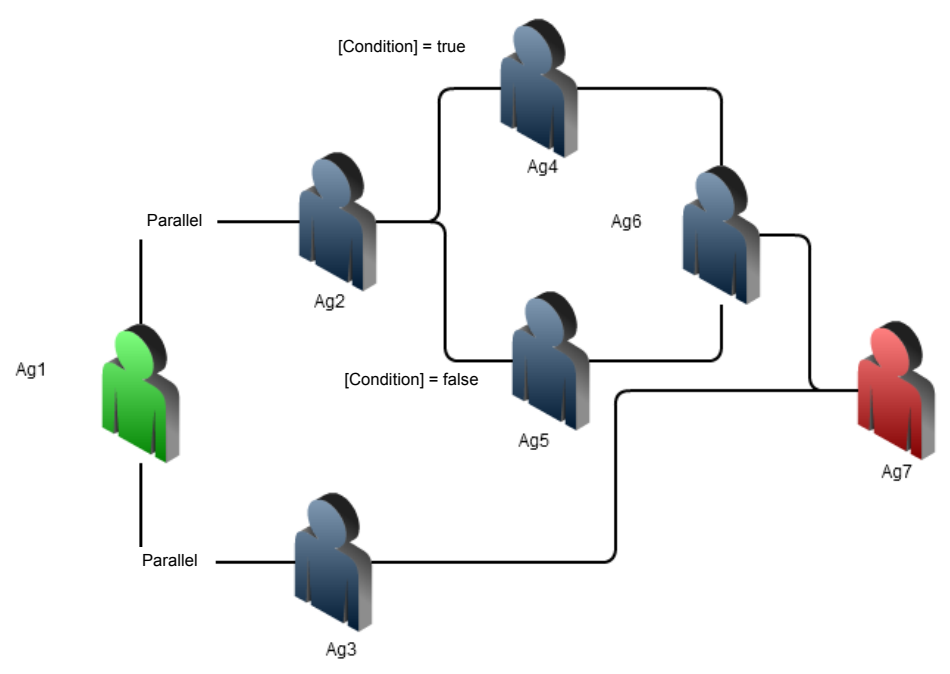

Figure A1: Agent subsystem representing a domain ontology built by the analysis system.

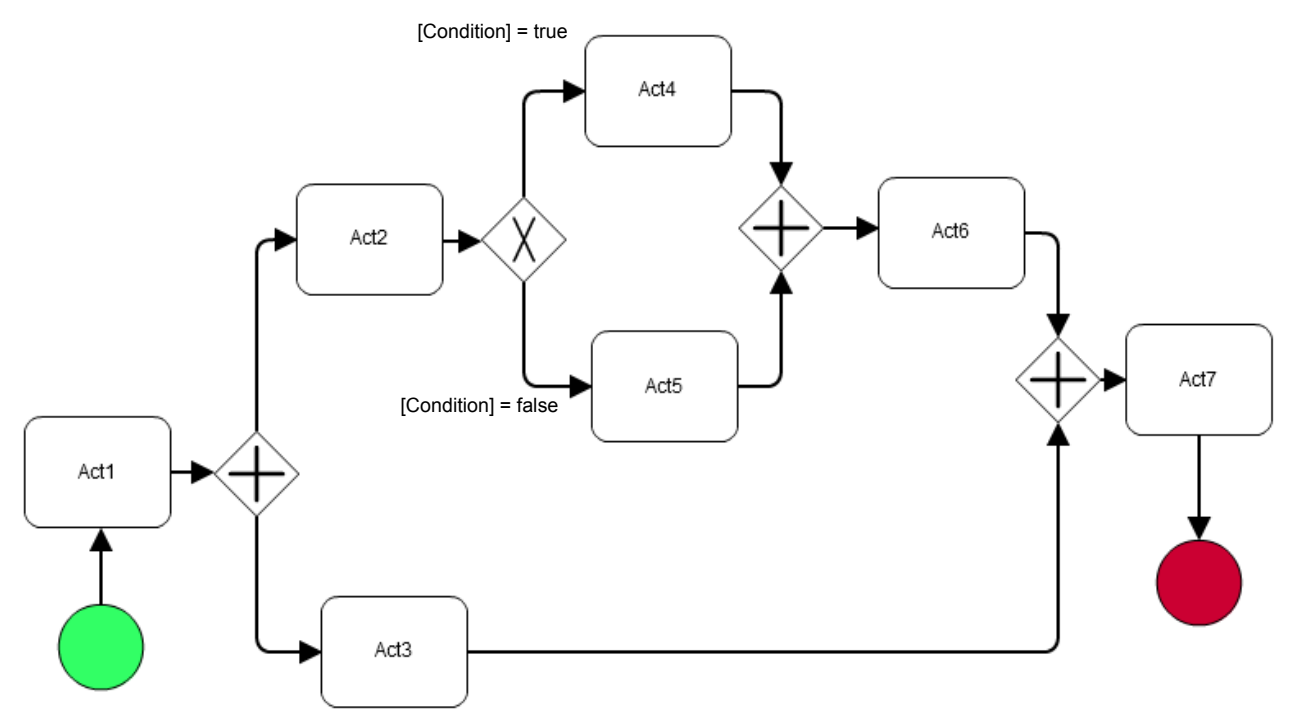

Figure A2: Diagram BPMN (BPD) mapped from the Agent subsystem in Figure A1.

Table A1: Semantics description of the attributes for modules CheckingRecord and NotifyState.

\begin{tabular}{rlr}
\hline Name: & CheckingRecord & Name: NotifyState \\
\hline Input: & Person & Input: person \\
& Organization & \\
Output: & recordState & Output:
\end{tabular}


Precondition: organizationExists

Action: verifyRecord

Domain: authentication

Type: startup
Precondition: personExists

Action: sendEmail

Domain: message

Type: end

Table A2: Semantics description of the attributes for modules CheckingBook and ChekingLoan.

\begin{tabular}{|c|c|c|c|}
\hline Name: & CheckingBook & Name: & ChekingLoan \\
\hline \multirow[t]{2}{*}{ Input: } & book & Input: & person \\
\hline & Organization & & organization \\
\hline Output: & bookState & Output: & loanState \\
\hline \multirow[t]{2}{*}{ Precondition: } & bookExists & Precondition: & personExists \\
\hline & organizationExists & & \\
\hline Action: & checkingAvailableBook & Action: & checkingLoan \\
\hline Domain: & Library & Domain: & library \\
\hline Type: & Intermediate & Type: & intermediate \\
\hline
\end{tabular}

Table A3: Semantics description of the attributes for modules NotifynoStock and NotifyLoanExceed.

\begin{tabular}{rlr}
\hline Name: & NotifynoStock & Name: NotifyLoanExceed \\
\hline Input: & person & Input: person \\
& book & \\
Output: & - & Output: \\
Precondition: & bookExists & Precondition: personExists \\
& personExists & \\
Action: & notifyNoBooks & Action: notifyNoLoan \\
Domain: & library & Domain: library \\
Type: & end & Type: end \\
\hline
\end{tabular}

Table A4: Semantics description of the attributes for module MakeLoan.

\begin{tabular}{cl}
\hline Name: & MakeLoan \\
\hline Input: & person
\end{tabular}




\begin{aligned} & \hline book \\ & Output: - \\ & Precondition: personExists \\ & bookExists \\ & Action: makeLoan \\ & Domain: library \\ & Type: End \\ & \hline\end{aligned}

Table A5: Semantics description of relationships Relation1 and Relation2 defined on the modules introduced by the user.

\begin{tabular}{rlrl}
\hline Name: & Relation1 & Name: & Relation2 \\
\hline Type: & If/Else & Type: Parallel \\
Startup: & CheckingRecord & Startup: CheckedRecord \\
Destination1: & NotifyState & Destination1: CheckingBook \\
Destination2: & CheckedRecord & Destination2: ChekingLoan \\
Condition: & registerState & Condition: & - \\
\hline
\end{tabular}

Table A6: Semantics description of relationships Relation3 and Relation4 defined on the modules introduced by the user.

\begin{tabular}{rlrl}
\hline Name: & Relation3 & Name: & Relation4 \\
\hline Type: & If/Else & Type: & If/Else \\
Startup: & CheckingBook & Startup: & ChekingLoan \\
Destination1: & MakeLoan & Destination1: & MakeLoan \\
Destination2: & NotifynoStock & Destination2: NotifyLoanExceed \\
Condition: & bookState & Condition: loanState \\
\hline
\end{tabular}

Table A7: Web services available in the platform for the case study of architecture IPCASCI.

\begin{tabular}{ll}
\hline Web services & Function \\
\hline $\begin{array}{l}\text { checkPersonRegister } \\
\text { checkOrgRegister }\end{array}$ & Check whether a user is registered in the system.
\end{tabular}


SendMail

sendEMail

emailManager

libraryManager

checkBook

compHLib

stockManager

loanManager

checkLoans

loanController

sendNotAvaliableBook

notifyBookError

loanPassed

notifyLoans

libraryLoanError

doLoan

libraryLoanEffect

bookStoreDoLoan
Send and manage the electronic mail (e-mail).

Identify books and manage their availability in the library.

Check and manage the (maximum) number of books loaned to a user.

Notify that there is not availability of a book or any other problem preventing the loan.

Notify that the maximum number of book loans has been reached.

They are used to register a book loan by the library.

Table A8: Web services found and checked by the search system for each module defined by the user.

\begin{tabular}{ll}
\hline Modules & Found web services \\
\hline CheckingRecord & $\begin{array}{l}\text { checkPersonRegister } \\
\text { checkOrgRegister }\end{array}$ \\
NotifyState & $\begin{array}{l}\text { sendMail } \\
\text { sendEMail }\end{array}$ \\
CheckingBook & compHLib \\
ChekingLoan & checkLoans \\
NotifynoStock & $\begin{array}{l}\text { sendNotAvaliableBook } \\
\text { notifyBookError } \\
\text { notifyLoans } \\
\text { libraryLoanError }\end{array}$ \\
\hline
\end{tabular}


Table A9: Web services selected for each defined module.

\begin{tabular}{ll}
\hline Activity/Module & Found web services \\
\hline CheckingRecord & checkPersonRegister \\
NotifyState & sendEMail \\
CheckingBook & compHLib \\
ChekingLoan & checkLoans \\
NotifynoStock & notifyBookError \\
NotifyLoanExceed & libraryLoanError \\
\hline
\end{tabular}

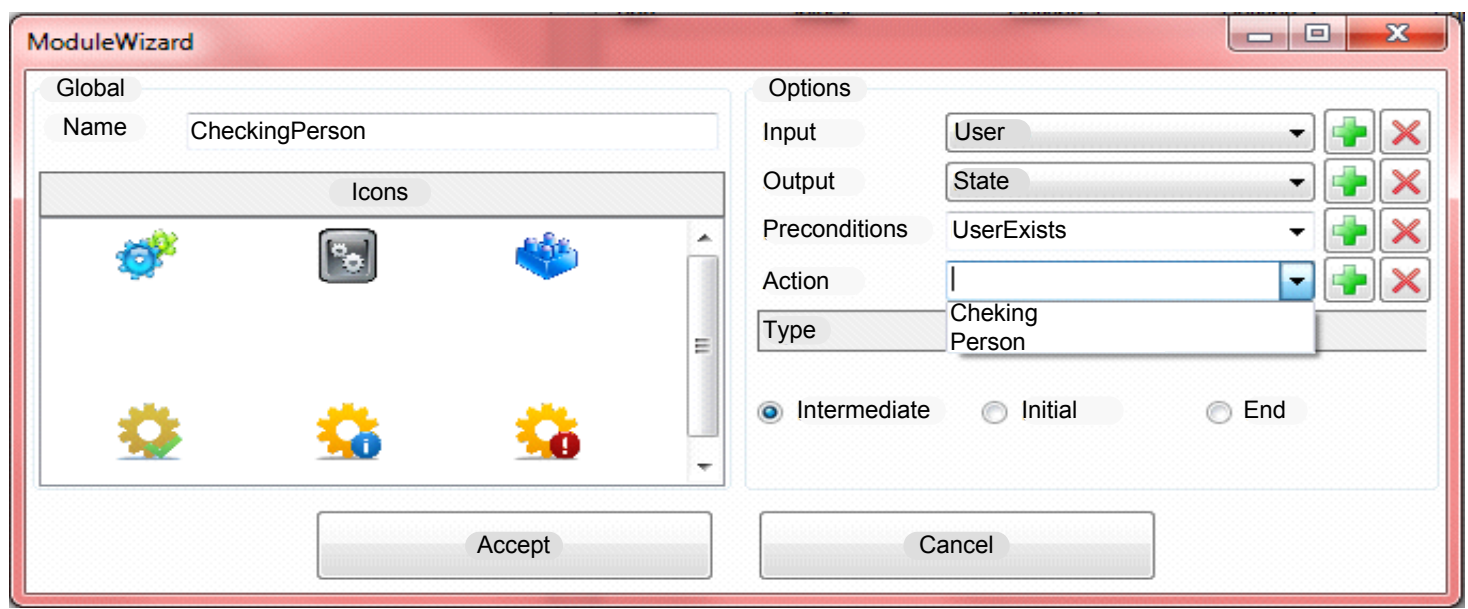

Figure A3: View of tool LibraryBookReserve showing an example to define a module and its semantics. 


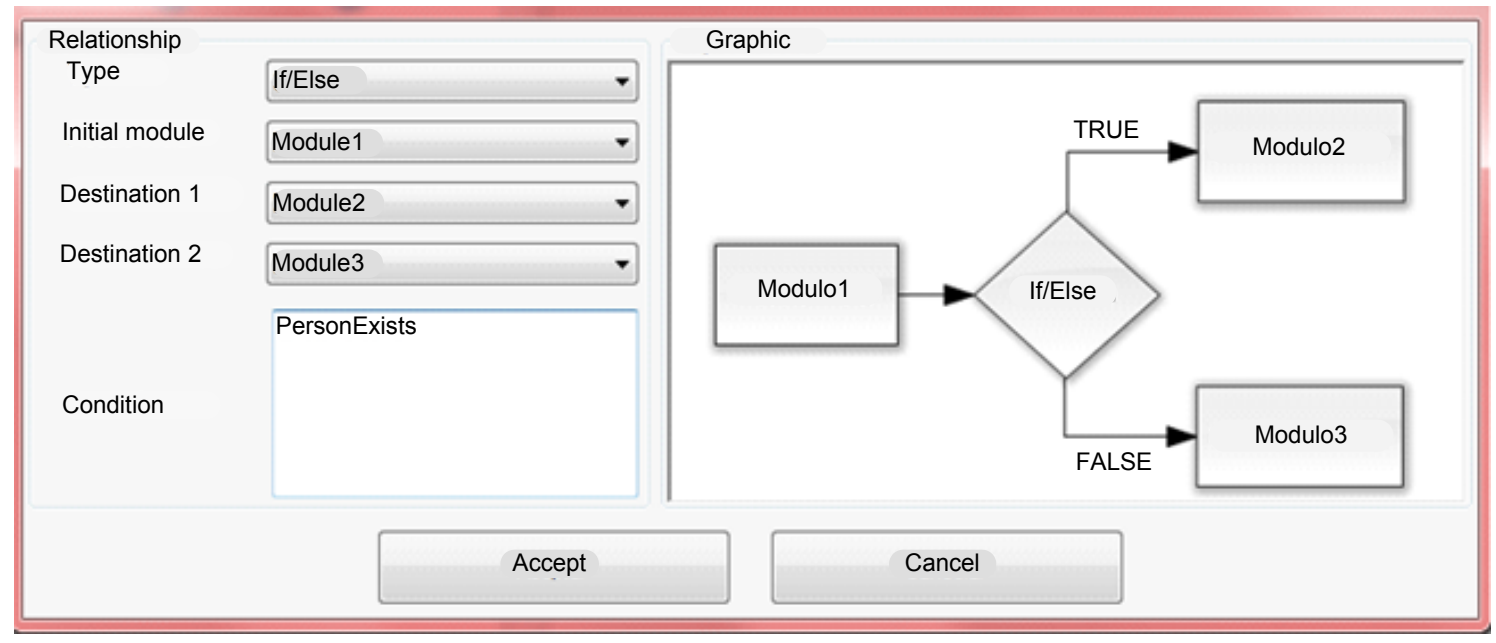

Figure A4: View of tool LibraryBookReserve showing an example to define the relationships between the built modules. 